COMMUNICATIONS IN

ANALYSIS AND GEOMETRY

Volume 5, Number 3, 439-474, 1997

\title{
Sharp Decay Estimates for Yang-Mills Fields
}

\section{DAVID Groisser ${ }^{1}$ AND Thomas H. PARKER ${ }^{2}$}

The instantons of Yang-Mills theory are localized solutions to global PDEs on a Riemannian 4-manifold $(M, g)$. As the instantons become more localized, their shape depends on the local geometry (around the point of concentration) and, to a lesser extent, on the global geometry of $M$. The relative importance of these effects is reflected in the geometry of the YangMills moduli space $\mathcal{M}(M)$ near its boundary. As one approaches the boundary, the solutions become more localized and simultaneously more like the standard solutions on the 4-sphere. Correspondingly, for $S U(2)$ connections (and presumably for other unitary connections) the geometry of the natural "Weil-Petersson" (or " $L$ ") metric on the moduli space becomes closer to the geometry of the Weil-Petersson metric on $\mathcal{M}\left(S^{4}\right)$. In this way, statements about the precise shape of instantons translate into statements about the geometry of moduli space.

The theorems of this paper give a variety of sharp pointwise upper bounds on the curvature of instantons. These yield new results about the geometry of moduli space. For example, they imply that for certain 4manifolds the sectional curvature near the boundary of the $S U(2) k=1$ moduli space is bounded, and simplify the proof that the boundary is totally geodesic in the $L^{2}$ completion $\overline{\mathcal{M}}$ regardless of the metric $g$ on $M$.

Pointwise bounds on curvature have been important in the analysis of Yang-Mills fields from the beginning. Uhlenbeck observed [U] that for any Yang-Mills connection $A$ and any point $x \in M$, the curvature $F_{A}$ of $A$ satisfies the pointwise bound

$$
\left|F_{A}\right|(y) \leq \frac{C(A, x)}{\operatorname{dist}(x, y)^{4}},
$$

where the constant $C(A, x)$ is independent of $y$ but potentially dependent upon $A$ and $x$. Many applications, however, require an estimate that is uniform in the connection. Donaldson [D] provided such an estimate for

\footnotetext{
${ }^{1}$ Partially supported by N.S.F. grant DMS-9307648

${ }^{2}$ Partially supported by N.S.F. grant DMS-9304013
} 
$k=1 S U(2)$ instantons: if the connection is centered at $x \in M$ and has scale $\lambda$ sufficiently small then

$$
\left|F_{A}\right|(y) \leq C \frac{\lambda^{2-\delta}}{\operatorname{dist}(x, y)^{4-\delta}}
$$

for all $y$ close to $x$. The $\delta$ in this bound, while rather unnatural, was introduced in Donaldson's proof to handle the fact that the metric is not euclidean in a neighborhood of $x$. Recently the first author showed that $(0.2)$ holds with $\delta=0$ provided the curvature of $(M, g)$ satisfies a certain positivity condition [G2]. There are also recent elegant results of Røade bounding general Yang-Mills fields on $\mathbb{R}^{4}[R]$. But the question remained whether one could get both the euclidean exponent $(\delta=0)$ and a constant $C$ independent of $A$ for a general manifold $(M, g)$. This question, we found, is related to the second derivatives of the metric at the boundary of moduli space (at least for $S U(2)$ connections).

The results proved here fall into two sets, roughly corresponding to improvements on the bounds $(0.1)$ and $(0.2)$ above. The results eliminate both of the problems mentioned above and show that on a general Riemannian 4-manifold one obtains estimates that have exactly the same form as on euclidean space; only the initial constant changes. In this sense our new bounds are "sharp".

Our first set of results concerns decay estimates for Yang-Mills fields on three types of domains: annuli of small outer radius, annuli near infinity on an asymptotically flat 4-manifold, and end regions of an asymptotically cylindrical manifold. In each case we bound the curvature for general YangMills fields, and show that better "one-sided" bounds hold when the connection is self-dual (SD) or anti-self-dual (ASD). In particular, we obtain several versions of (0.1) on that hold, on annuli $\Omega$, with a constant independent of $x$ and whose only dependence on $A$ is through the energy $\left\|F_{A}\right\|_{L^{2}(\Omega)}^{2}$. These bounds are stated in section 1 and proved in sections 2 and 3 . The key to the proofs is keeping careful track of the metric as one works through the required eigenvalue estimates and some rather tricky maximum principle arguments. It is enough to establish these bounds on one of the three domains mentioned above; the corresponding bounds for the other domains then follow by conformal invariance.

Specifically, in section 2 we work with SD/ASD connections on an asymptotically cylindrical 4-manifold, and follow Donaldson's original argument [D]. The new ingredient here is the observation that a simple eigenvalue estimate (Lemma 2.2) obviates the need for the $\delta$ in (0.2). 
In section 3 we work with general Yang-Mills fields on an asymptotically flat manifold, adapting the comparison principle argument of Røade $[R]$ to the case of non-constant metric. Whereas Røade compares $\left|F_{A}\right|$ with powers of the distance function, we compare it with powers of the Green's function of the laplacian, and then bound the Green's function. The required pointwise bounds on the Green's function (which unfortunately do not appear in the literature on asymptotically flat manifolds) are derived in the appendix.

Our second set of results focuses on unitary SD/ASD connections near the boundary of moduli space. Such connections consist of a smooth "background" connection with one or more sharply concentrated "instantons" superimposed on it. In section 4 we first make precise the notion of concentrated connections and show that these indeed form a neighborhood of the boundary of moduli space. We then obtain separate bounds on $|F|$ in (i) the regions close to the instantons, (ii) the intermediate collars around the instantons, and (iii) the regions far from the instantons. All of these estimates involve sharp exponents, that is, they correspond to $\delta=0$ in (0.2) . Moreover, for connections near the "bottom stratum" of moduli space (where the background connection is trivial and each concentrated instanton has charge 1 ) we obtain a global estimate that generalizes $(0.2)$ to higher instanton number and general 4-manifolds (Theorem 4.7). The main technical difficulty in the proofs of section 4 is ensuring that the estimates are uniform for families of instantons where the points of concentration are coalescing.

Section 5 gives applications to the geometry of the SD/ASD $S U(2)$ moduli space $\mathcal{M}$ near its boundary. Previous work by the authors [G1],[G2], [GP1],[GP2] and P. Feehan [F] has yielded a good understanding of the $C^{0}$ properties of this metric; for example, the metric is incomplete, so the Cauchy completion $\overline{\mathcal{M}}$ has nonempty boundary $\partial \mathcal{M}$. In certain cases this boundary is particularly simple - an isometric copy of $M$ itself. But while the authors cited above were able to draw no conclusions about properties involving derivatives of the metric on general $S U(2)$ moduli spaces $\overline{\mathcal{M}}$, calculations of concrete examples $\left(k=1\right.$ and $M=S^{4}$ or $\left.\mathbf{C} P^{2}\right)$ suggested that the first few derivatives might in fact be very well behaved at the boundary. In section 5 we show that the estimates of section 4 yield $C^{1}$ and $C^{2}$ statements about the geometry of the moduli space $\mathcal{M}_{1}$ of $k=1 S U(2)$ instantons over a simply-connected definite four-manifold $M$ with any smooth metric $g$, near the boundary of $\mathcal{M}_{1}$ : (i) the $L^{2}$ metric extends to a $C^{1}$ metric on the completion $\overline{\mathcal{M}}$, (ii) the boundary $\partial \overline{\mathcal{M}}$ is totally geodesic, and (iii) the sectional curvature of $\mathcal{M}$ is bounded near $\partial \overline{\mathcal{M}}$. The new estimates of this paper are needed to prove (iii) in the stated generality, and simplify the 
proofs of (i) and (ii).

Finally, we mention the connection with physics. The $L^{2}$ metric on the space of connections is important in physical Yang-Mills theory because its associated measure is the one that physicists (implicitly) use for path integrals. In fact, the expansion of the metric at the boundary of moduli space is closely related to the "beta function expansions" and the notion of "asymptotic freedom" that play an important role in the physics of YangMills fields. The relation between these physical quanties and the expansion of the metric on the $S U(2)$ moduli space of the standard 4-sphere was described in [GP3]. The results of section 5 show that significant parts of that discussion carry over to arbitrary metrics on $S^{4}$.

\section{General Decay Bounds.}

Our first set of results take the form of three theorems that give pointwise bounds on the curvature of Yang-Mills fields on various types of Riemannian 4-manifolds $(M, g)$. These are described here and proven in sections 2 and 3 .

Below, $\|\cdot\|_{\Omega}$ denotes the $L^{2}$ norm taken over a region $\Omega \subset l M$. We use $B(p, s)$ to denote the closed geodesic ball of radius $s$ centered at $p \in M$, and $\Omega\left(p, r_{0}, r_{1}\right)$ to denote the closed annulus $\overline{B\left(p, r_{1}\right)-B\left(p, r_{0}\right)}$. We often fix $p$ and let $r$ denote distance to $p$. The curvature of a connection $A$ is denoted $F_{A}$ (or just $F$ ), and the energy in a region $\Omega$ is

$$
E(\Omega):=\frac{1}{8 \pi^{2}} \int_{\Omega}\left|F_{A}\right|^{2} d v_{\tilde{g}}
$$

Of course, when discussing self-dual (SD) or anti-self-dual (ASD) connections we assume that $M$ is oriented.

The first theorem gives local decay estimates that apply when $(M, g)$ is compact or, more generally, has "bounded geometry" (positive injectivity radius and curvature bounded in $C^{2}$ ).

Theorem 1.1. Let $(M, g)$ be a Riemannian manifold of bounded geometry and let $P \rightarrow M$ be a principal $G$-bundle with $G$ compact. There exist constants $C, \varepsilon_{0}, \rho>0$, depending on $(M, g)$ and $G$, such that if $0 \leq 4 r_{0} \leq r_{1} \leq \rho$ and $A$ is a Yang-Mills connection on $\Omega=\Omega\left(p, r_{0}, r_{1}\right)$ with $\left\|F_{A}\right\|_{\Omega}^{2} \leq \varepsilon_{0}$, then

$$
\left|F_{A}(y)\right| \leq C\left(\frac{r_{0}^{2}}{r^{4}}+\frac{1}{r_{1}^{2}}\right)\left\|F_{A}\right\|_{\Omega} \quad \text { for } y \in \Omega\left(p, 2 r_{0}, r_{1} / 2\right) .
$$


Moreover, if $A$ is a $S D$ or $A S D$ unitary connection on $B\left(p, r_{1}\right)$ with $\left\|F_{A}\right\|_{\Omega}^{2} \leq$ $\varepsilon_{0}$ then

(a) If $k \leq E\left(B\left(p, r_{0}\right)\right)<k+\frac{1}{2}$ for some $k \in \mathbb{Z}$ then

$$
\left|F_{A}(y)\right| \leq \frac{C}{r_{1}^{2}}\left\|F_{A}\right\|_{\Omega} \quad \text { for } y \in \Omega\left(p, 2 r_{0}, r_{1} / 2\right) .
$$

(b) If $k-\frac{1}{2}<E\left(B\left(p, r_{1}\right)\right) \leq k$ for some $k \in \mathbb{Z}$ then

$$
\left|F_{A}(y)\right| \leq C \frac{r_{0}^{2}}{r^{4}}\left\|F_{A}\right\|_{\Omega} \quad \text { for } y \in \Omega\left(p, 2 r_{0}, r_{1} / 2\right) .
$$

At the end of this section we comment on the distinction between cases (a) and (b). Letting $r_{0} \rightarrow 0$ in (1.2) shows that $|F|$ is bounded on $B\left(p, r_{1}\right)-$ $\{p\}$; this leads easily to a proof of the removable singularities theorem for YM fields (cf. [FU]).

We can similarly describe the decay of Yang-Mills fields "at infinity". For this we assume that $(M, g)$ is an asymptotically flat 4-manifold in the sense used in general relativity. This means that $M$ is a disjoint union $M_{0} \cup M_{\infty}$, where $M_{0}$ is compact and for some $R_{0}>0$ there is a diffeomorphism of $M_{\infty}$ with $\mathbb{R}^{4}-B\left(0, R_{0}\right)$ giving coordinates $\left\{x^{i}\right\}$ in which the metric has the form

$$
g_{i j}=\delta_{i j}+h_{i j}
$$

with $r^{2}\left|h_{i j}\right|+r^{3}\left|\partial_{k} h_{i j}\right|+r^{4}\left|\partial_{k} \partial_{l} h_{i j}\right| \leq C(g)$ for some constant $C(g)$ and $r=|x|$. We then get a decay estimate as $r \rightarrow \infty$ that is entirely analogous to Theorem 1.1. In this theorem, $\Omega\left(r_{0}, r_{1}\right)$ denotes the annulus $r_{0} \leq r \leq r_{1}$ in $M_{\infty}$, and $E(r, \infty)$ denotes the energy $E(\Omega(r, \infty))$.

Theorem 1.2. Let $(M, g)$ be an asymptotically flat 4-manifold, and let $G, P$ be as in Theorem 1.1. There exists constants $C, \varepsilon_{0}>0$ and $R \geq R_{0}$, depending on $C(g)$ and $G$, such that if $4 R_{0} \leq 4 r_{0} \leq r_{1} \leq \infty$ and $A$ is a Yang-Mills connection on $\Omega=\Omega\left(r_{0}, r_{1}\right)$ with $\|F\|_{\Omega}^{2} \leq \varepsilon_{0}$ then

$$
|F(y)| \leq C\left(\frac{r_{0}^{2}}{r^{4}}+\frac{1}{r_{1}^{2}}\right)\|F\|_{\Omega} \quad \text { for } y \in \Omega\left(2 r_{0}, r_{1} / 2\right) .
$$

Moreover, if $A$ is a $S D$ or $A S D$ unitary connection on $\Omega\left(r_{0}, \infty\right)$ with $\left\|F_{A}\right\|_{\Omega}^{2} \leq \varepsilon_{0}$ then 
(a) If $k \leq E\left(r_{1}, \infty\right)<k+\frac{1}{2}$ for some $k \in \mathbb{Z}$ then

$$
|F(y)| \leq C \frac{r_{0}^{2}}{r^{4}}\|F\|_{\Omega} \quad \text { for } y \in \Omega\left(2 r_{0}, r_{1} / 2\right),
$$

(b) If $k-\frac{1}{2}<E\left(r_{0}, \infty\right) \leq k$ for some $k \in \mathbb{Z}$ then

$$
|F(y)| \leq \frac{C}{r_{1}^{2}}\|F\|_{\Omega} \quad \text { for } y \in \Omega\left(2 r_{0}, r_{1} / 2\right) .
$$

Letting $r_{1} \rightarrow \infty$ in (1.6) shows that a finite-energy Yang-lMills field decays as $O\left(1 / r^{4}\right)$.

We can also consider the case of an asymptotically cylindrical manifold. Here $M=M_{0} \cup M_{\infty}$ is a disjoint union where $M_{0}$ is compact ancl for some $T_{0} \geq 0$ there is a diffeomorphism of $M_{\infty}$ with $\left[T_{0}, \infty\right) \times S^{3}$ giving ccordinates $\{t, y\}$ in which the metric has the form

$$
g=d t^{2}+g_{0}+h,
$$

where $g_{0}$ is the standard metric on $S^{3}$ and $h$ is a 2-tensor on $\left[T_{0}, \infty\right) \times S^{3}$ satisfying $\left|h_{i j}\right|+\left|\partial_{k} h\right|+\left|\partial_{k} \partial_{l} h\right| \leq C(g) e^{-2 t}$. Write $E(t, \infty)$ for the energy in $[t, \infty] \times S^{3}$.

Theorem 1.3. Let $(M, g)$ be an asymptotically cylindrical 4-manifold, and let $G, P$ be as in Theorem 1.1. There exists constants $C, \varepsilon_{0}>0$ and $T \geq T_{0}$, depending on $C(g)$ and $G$, such that if $T \leq t_{0} \leq t_{1}-2 \leq \infty$ and $A$ is a Yang-Mills connection on $\Omega=\left[t_{0}, t_{1}\right] \times S^{3}$ with $\|F\|_{\Omega}^{2} \leq \varepsilon_{0}$ then

$$
\begin{aligned}
|F(y)| \leq C\left(e^{-2\left(t-t_{0}\right)}+e^{-2\left(t_{1}-t\right)}\right) & \|F\|_{\Omega} \\
& \text { for } y \in\left[t_{0}+1, t_{1}-1 .\right] \times S^{3} .
\end{aligned}
$$

Moreover, if $A$ is a $S D$ or $A S D$ unitary connection on $\left[t_{0}, \infty\right] \times S^{3}$ with $\left\|F_{A}\right\|_{\Omega}^{2} \leq \varepsilon_{0}$ then

(a) If $k \leq E\left(t_{1}, \infty\right)<k+\frac{1}{2}$ for some $k \in \mathbb{Z}$ then

$$
|F(y)| \leq C e^{-2\left(t-t_{0}\right)}\|F\|_{\Omega} \quad \text { for } y \in\left[t_{0}+1, t_{1}-1\right] \times S^{3},
$$

(b) If $k-\frac{1}{2}<E\left(t_{0}, \infty\right) \leq k$ for some $k \in \mathbb{Z}$ then

$$
|F(y)| \leq C e^{-2\left(t_{1}-t\right)}\|F\|_{\Omega} \quad \text { for } y \in\left[t_{0}+1, t_{1}-1\right] \times S^{3} .
$$


Each of these theorems makes a stronger statement for SD connections than for general Yang-Mills fields. This is because SD connections satisfy a first order equation, so their values on an annulus $\Omega$ can be bounded in terms of their values on one component of $\partial \Omega$. Bounding a solution of the second order Yang-Mills equations, on the other hand, necessarily involves the values on both components of $\partial \Omega$.

In bounding SD and ASD fields, each of these theorems distinguishes two cases. This distinction can be understood as follows. Consider a self-dual connection $A$ on the end of an asymptotically cylindrical manifold. The energy density $\left|F_{A}\right|^{2}$ may separate the end into a finite number of regions containing instanton "bumps", each with integral energy, lying between values $t=T_{k}$ where $E\left(T_{k}, \infty\right)=k \in \mathbb{Z}$. Theorem 1.3a bounds the energy density in a region where it is decreasing as $t$ increases (e.g. region $\mathrm{I}$ in Figure 1), while Theorem $1.3 \mathrm{~b}$ bounds the energy density in a region where it is increasing (e.g. region II in Figure 1). The interpretation of cases (a) and (b) in Theorems 1.1 and 1.2 is similar. Thus these theorems give decay rates not just outside instantons, but also between instantons.

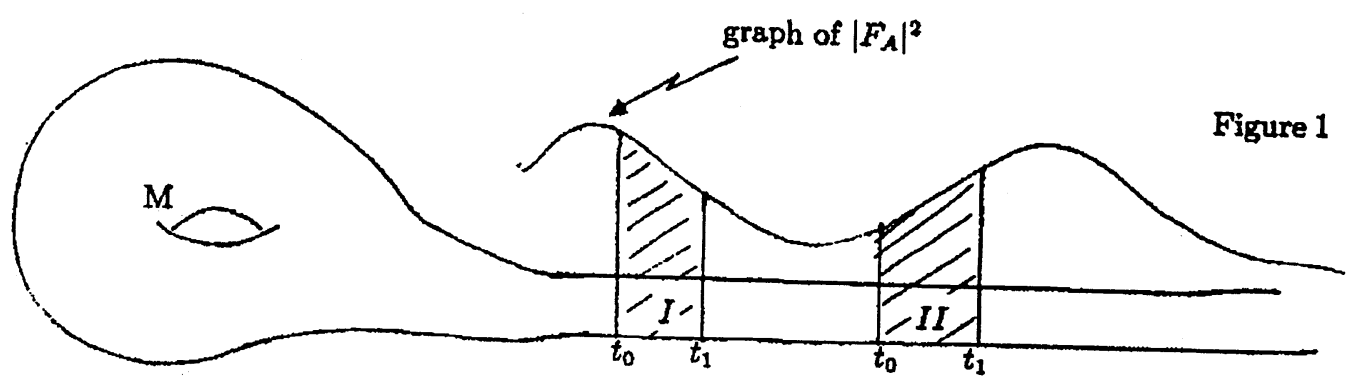

Theorems 1.1-1.3 are related by conformal diffeomorphisms. In section 2 we prove Theorem 1.3ab and show how to use conformal transformations to deduce Theorem 1.1ab and Theorem 1.2ab. In section 3 we consider general Yang-Mills fields, this time proving (1.6), and then obtaining (1.2) and (1.10) by conformal transformations.

\section{Self-Dual/ Anti-Self-Dual Fields.}

In this section we consider $\mathrm{SD} / \mathrm{ASD} U(n)$-connections on a rank $n$ hermitian bundle $E$ on an asymptotically cylindrical manifold $(M, g)$ and prove the decay estimates (1.11) and (1.12) of Theorem 1.3. 
The proof is based on the properties the Chern-Simons functional. Specifically, given a connection form (an $\operatorname{End}(E)$-valued 1-form), we define the Chern-Simons 3-form

$$
C S(a)=\operatorname{tr}\left(a \wedge d a+\frac{2}{3} a \wedge a \wedge a\right) .
$$

If $a$ is a connection form (determined by some gauge choice) for a connection $A$ with curvature $F_{A}$, then $d C S(a)=\operatorname{tr}\left(F_{A} \wedge F_{A}\right)$. Hence for a SD/ASD connection on a compact domain $D \subset M$ over which there exists a global connection form $a$, the energy in $D$ is

$$
E(D)=\frac{1}{8 \pi^{2}} \int_{D}\left|F_{A}\right|^{2}=-\frac{1}{8 \pi^{2}} \int_{D} \operatorname{tr}\left(F_{A} \wedge * F_{A}\right)=\mp \frac{1}{8 \pi^{2}} \int_{\partial D} C S(a)
$$

(here and below the top sign is for SD connections, the bottom for ASD) . The normalization factor $1 / 8 \pi^{2}$ is choosen so that $E(M)$ is integral - it is the characteristic number $c_{2}(E)-\frac{1}{2} c_{1}^{2}(E)$.

A basic property of the Chern-Simons integrand is that, given any two connection forms for the same connection on a fixed oriented three-manifold, the corresponding Chern-Simons integrals may differ as real nurabers, but agree modulo $\mathbb{Z}$. Since any four-dimensional compact domain $D \subset M$ can be decomposed into subdomains over which a given bundle $P$ is trivial, it follows that

$$
E(D) \equiv \mp \frac{1}{8 \pi^{2}} \sum_{i} \int_{(\partial D)_{i}} C S\left(a_{i}\right) \bmod \mathbb{Z}
$$

for any connection forms $a_{i}$ representing $A$ on the components $(\partial D)_{i}$ of the boundary $\partial D$.

We apply this to a connection $A$ on the cylinder $\left[t_{0}, t_{1}\right] \times S^{3}$ that satisfies the hypotheses of Theorem 1.3. Since $E$ is a $U(n)$-bundle it is trivial over each 3-sphere $S_{t}=\{t\} \times S^{3}$, so we can choose a gauge near each $S_{t}$, with corresponding connection form $a_{t}$. Thus if we set

$$
J(t)= \pm \frac{1}{8 \pi^{2}} \int_{S_{t}} C S\left(a_{t}\right)
$$

we have

$$
E(s, t):=E\left([s, t] \times S^{3}\right) \equiv J(s)-J(t) \quad \bmod \mathbb{Z} .
$$


At the moment, the $a_{t}$ can vary arbitrarily (even discontinuously) with $t$; we will restrict the choice of $a_{t}$ momentarily. Below, we will write $g_{t}$ for the metric induced on $S_{t}$; these are uniformly equivalent to the standard metric for $t \geq T$. Also, given a 1-form $a$ on a neighborhood of the slice $S_{t}$, we let $\bar{a}_{t}$ denote the pullback of $a$ to $S_{t}$, and $\bar{F}_{t}$ the corresponding curvature form on $S_{t}$. As in Theorem 1.3 we assume that the energy $E:=E\left(\left[t_{0}, t_{1}\right] \times S^{3}\right)$ is at most $\varepsilon_{0}$ (to be determined below).

If we take $\varepsilon_{0}$ small enough, Uhlenbeck's sup bound ([FU] Theorem 8.3) gives

$$
\sup _{S_{t}}|F|^{2} \leq c_{1} \int_{\left[t-\frac{1}{2}, t+\frac{1}{2}\right] \times S^{3}}|F|^{2} \leq c_{1} E \leq c_{1} \varepsilon_{0} .
$$

for $t$ in the interval $\left[t_{0}^{\prime}, t_{1}^{\prime}\right]=\left[t_{0}+\frac{1}{2}, t_{1}-\frac{1}{2}\right]$. Because the connection is SD or ASD its curvature satisfies $|F|^{2}=2|\bar{F}|^{2}$ and hence

$$
\int_{S_{t}}\left|\bar{F}_{t}\right|^{2} \leq c_{2} E \leq c_{2} \varepsilon_{0}
$$

A second result of Uhlenbeck (see [DK, Proposition 4.4.11]) shows that when (2.7) holds with $\varepsilon_{0}$ small there exists a gauge near $S_{t}$ whose corresponding connection form $a_{t}$ satisfies

$$
\left\|d \overline{a_{t}}\right\|_{L^{2}\left(S_{t}\right)}^{2}+\left\|\overline{a_{t}}\right\|_{L^{3}\left(S_{t}\right)}^{3}+\left\|\overline{a_{t}}\right\|_{L^{4}\left(S_{t}\right)}^{4} \leq c_{3} \int_{S_{t}}\left|\bar{F}_{t}\right|^{2} \leq c_{2} c_{3} E
$$

(these constants $c_{2}, c_{3}$ can be taken independent of $t$ since the induced metrics on $S_{t}$ are uniformly equivalent by (1.9)). Henceforth we choose such a gauge for each $t \in\left[t_{0}^{\prime}, t_{1}^{\prime}\right]$. It follows easily the Chern-Simons function (2.4) then satisfies

$$
|J(t)| \leq c_{4} E \quad \text { for } t \in\left[t_{0}^{\prime}, t_{1}^{\prime}\right]
$$

and that for $t \in\left[t_{0}^{\prime}, t_{1}^{\prime}\right](2.5)$ holds as an equality in $\mathbb{R}$, not just $\bmod \mathbb{Z}$.

It is important to distinguish the sign of $J(t)$. Indeed, this is what separates the two cases of Theorem 1.3. Since $E\left([t-1, t+1] \times S^{3}\right) \rightarrow 0$ as $t \rightarrow \infty$, the argument leading to (2.9) shows that we can choose gauges to arrange $\lim _{t \rightarrow \infty} J(t)=0$. Hence, using (2.5), for each $t \in\left[t_{0}, \infty\right)$ there is an integer $k_{t}$ such that $J(t)=E(t, \infty)-k_{t}$, and when $t \in\left[t_{0}^{\prime}, t_{1}^{\prime}\right](2.9)$ implies that $k_{t}$ is the unique integer $k$ (independent of $t$ ) such that $|E(t, \infty)-k| \leq$ 
$c_{4} E<1 / 2$. We extend our gauge choices over $\left(\left[t_{0}, t_{0}^{\prime}\right] \cup\left[t_{1}^{\prime}, t_{1}\right]\right) \times S^{3}$ to make $J(t)$ continuous on $\left[t_{0}, t_{1}\right] \times S^{3}$. It then follows that for all $s, t \in\left[t_{0}, t_{1}\right]$, (2.5) holds as in equality in $\mathbb{R}$ and that $J(t)=E(t, \infty)-k$. Consequently, the hypotheses of Theorem $1.3 \mathrm{a}$ imply that $J\left(t_{1}\right) \geq 0$, and the hypotheses of Theorem 1.3b imply that $J\left(t_{0}\right) \leq 0$. Thus Theorem 1.3ab is a direct consequence of the next proposition, which establishes the decay rates of Theorem 1.3 without assuming that the connection extends to infinity.

Proposition 2.1. Let $(M, g)$ be an asymptotically cylindrical 4-manifold, and let $G, P$ be as in Theorem 1.3 with $G$ a unitary group. Given a connection on $P$, define $J(t) \in \mathbb{R} / \mathbb{Z}$ by (2.4). There exists constants $T, C, \varepsilon_{0}>0$, depending on $C(g)$ and $G$, such that any $S D$ or $A S D$ connection $A$ on $\Omega=\left[t_{0}, t_{1}\right] \times S^{3}$ with $T \leq t_{0} \leq t_{1}-2$ and $\|F\|_{\Omega}^{2} \leq \varepsilon_{0}$ then

(a) If $J\left(t_{1}\right) \in[0,1 / 2) \bmod \mathbb{Z}$ then

$$
|F(y)| \leq C e^{-2\left(t-t_{0}\right)}\|F\|_{\Omega} \quad \text { for } y \in\left[t_{0}+1, t_{1}-1\right] \times S^{3},
$$

(b) If $J\left(t_{0}\right) \in(-1 / 2,0] \bmod \mathbb{Z}$ then

$$
|F(y)| \leq C e^{-2\left(t_{1}-t\right)}\|F\|_{\Omega} \quad \text { for } y \in\left[t_{0}+1, t_{1}-1\right] \times S^{3} .
$$

Following Donaldson [D], we will prove this by establishing a differential inequality for the derivative $J^{\prime}$. The key ingredient is the eigenvalue estimate in the following lemma.

Lemma 2.2. There exists a constant $B$ such that for any 1-form $\omega$ on $\left(S_{t}, g_{t}\right)$ we have

$$
\left|\int_{S_{t}} \omega \wedge d \omega\right| \leq \frac{1}{\sqrt{\mu_{t}}} \int_{S_{t}}|d \omega|_{g_{t}}^{2} d v_{g_{t}}
$$

where for $t \geq T_{0}$

$$
\left|4-\mu_{t}\right| \leq B e^{-2 t}
$$

Proof. It follows easily from Hölder's inequality and the Hodge theorem that (2.13) holds with $\mu_{t}$ equal to the first positive eigenvalue of the Laplacian on 
coclosed 1-forms on $\left(S_{t}, g_{t}\right)$. For the standard metric on $S^{3}$ we have $\mu=4$ (as in [DK] Lemma 7.3.4). In general

$$
\mu_{t}=\inf _{d^{*} \omega=0} \frac{\int|d \omega|^{2}}{\int|\omega|^{2}}
$$

where the norms are with respect to $g_{t}$. Writing $\omega=* \phi$ where $\phi$ is a 2-form, this becomes

$$
\mu_{t}=\inf _{d \phi=0} \frac{\int|d * \phi|^{2}}{\int|\phi|^{2}}:=E\left(\phi, g_{t}\right) .
$$

The dependence on the metric in this last expression is purely algebraic, so

$$
(1-c|h|) E\left(\phi, g_{t}\right) \leq E\left(\phi, g_{0}\right) \leq(1+c|h|) E\left(\phi, g_{t}\right) .
$$

Taking the infimum over all closed 2-forms $\phi$ and using (1.9) shows that

$$
\left|\mu_{t}-4\right| \leq c|h(t)| \leq B e^{-2 t} .
$$

It is easy to check that the same constant $\mu_{t}$ works for $\operatorname{End}(E)$-valued 1-forms $\omega$ after replacing $\omega \wedge d \omega$ by $\operatorname{tr}(\omega \wedge d \omega)$ in (2.12)).

Remark. Our use of the strong decay in (2.13) is what distinguishes our proof of the SD/ASD decay estimates from those in [D] and [G2]. In his original proof Donaldson observed that $\mu_{t}$ could be taken arbitrarily close to 4 , so he replaced the right-hand side of (2.13) by an arbitrary $\delta>$ 0 , and derived (0.2) by essentially the same argument we give below. In [G2] the first author noted that, under a certain positivity condition on the Riemannian curvature, one still has $\lim \inf _{t \rightarrow \infty} \mu_{t} \geq 4$, and that this is enough to establish (0.2) with $\delta=0$. The proof below shows that the curvature condition is a red herring-because of (2.13), $\mu_{t}$ approaches 4 so fast that it doesn't matter whether $\mu_{t}>4$ or $\mu_{t}<4$; one gets the same sharp exponent as one would if $\mu_{t}$ were identically 4 .

With these facts in hand, we turn to the proof of Proposition 2.1a,b. Cases (a) and (b) are parallel but slightly different. In both cases, we lift $J(t)$ to a real-valued function on $\left[t_{0}, t_{1}\right]$ using the gauge choices discussed earlier, so that (2.5) holds in $\mathbb{R}$ (not just $\mathbb{R} / \mathbb{Z}$ ) for $s, t \in\left[t_{0}, t_{1}\right]$. Also $t_{0}^{\prime}, t_{1}^{\prime}$ are as in our earlier discussion. 
Case (a) When $J\left(t_{1}\right) \geq 0$, we set $E(t)=E\left(t, t_{1}\right)$. Then by (2.5) and (2.9), for all $t \in\left[t_{0}, t_{1}\right]$ we have

$$
0 \leq E(t)=J(t)-J\left(t_{1}\right) \leq J(t) \leq c_{4} E \leq c_{4} \varepsilon_{0}
$$

and, again noting that $|F|=2\left|\bar{F}_{t}\right|$ on $S_{t}$,

$$
8 \pi^{2} J^{\prime}=8 \pi^{2} E^{\prime}=-2 \int_{S_{t}}\left|\bar{F}_{t}\right|_{g_{t}{ }^{1} \partial / \partial t} d v_{g} \leq 0
$$

The volume forms $1_{\partial / \partial t} d v_{g}$ and $d v_{g_{t}}$ are generally not equal, but by (1.9) their ratio is $1+O\left(e^{-2 t}\right)$. Hence for large $t$

$$
8 \pi^{2} J^{\prime} \leq-2\left(1-c_{5} e^{-2 t}\right) \int_{S^{3}}\left|d \overline{a_{t}}+\overline{a_{t}} \wedge \bar{a}_{t}\right|_{g_{t}}^{2} d v_{g_{t}} .
$$

Expanding the integrand, applying Lemma 2.2 and Hölder's inequality,

$$
\begin{aligned}
8 \pi^{2} J^{\prime} \leq & -2\left(1-c_{5} e^{-2 t}\right) \sqrt{\mu_{t}}\left|\int_{S_{t}} C S\left(\overline{a_{t}}\right)\right| \\
& +2\left(\sqrt{\mu_{t}}\left\|\overline{\bar{t}_{t}}\right\|_{3}^{3}+\left\|d \overline{a_{t}}\right\|_{2}\left\|\overline{a_{t}}\right\|_{4}^{2}+\left\|\overline{a_{t}}\right\|_{4}^{4}\right)
\end{aligned}
$$

where these norms taken with respect to $g_{t}$. Using (2.13), (2.8) and (2.15) this simplifies to

$$
J^{\prime} \leq-4\left(1-c_{6} e^{-2 t}\right)|J|+c_{7}\left|J^{\prime}\right|^{3 / 2}
$$

with $J^{\prime}<0$. Writing $x=|J|$ and $y=-J^{\prime}$, this has the form $y \geq a x-b y^{3 / 2}$ with $a \leq 4$. By elementary algebra this implies that $y \geq a x-8 b x^{3 / 2}$ (write $y=\lambda x$ and consider the cases $\lambda \geq a$ and $\lambda \leq a$ ). Therefore

$$
J^{\prime} \leq\left(-4+4 c_{6} e^{-2 t}+8 c_{7}|J|^{1 / 2}\right)|J| \quad \text { for } t_{0}^{\prime} \leq t \leq t_{1}^{\prime}
$$

We will integrate this twice. For this we impose the conditions that $T$ is large enough and $\varepsilon_{0}$ are small enough that

$$
4 c_{6} e^{-2 T} \leq 1 \quad \text { and } \quad c_{4} \varepsilon_{0} \leq \frac{1}{64 c_{7}^{2}}
$$

Then (2.14) and (2.16) give $J^{\prime} \leq-2 J$ as well as $J \geq 0$, so $J(t) \leq$ $J\left(t_{0}^{\prime}\right) e^{-2\left(t-t_{0}^{\prime}\right)}$ with $J\left(t_{0}^{\prime}\right) \leq\left(8 c_{7}\right)^{-2}$ by $(2.14)$ and (2.17). Putting this back into (2.16) and integrating,

$$
\log \frac{J(t)}{J\left(t_{0}^{\prime}\right)} \leq\left.\left(-4 t-2 c_{6} e^{-2 t}-e^{-\left(t-t_{0}^{\prime}\right)}\right)\right|_{t_{0}^{\prime}} ^{t} \leq-4\left(t-t_{0}^{\prime}\right)+\beta
$$


with $\beta \leq 3 / 2$ by (2.17). Combining this with (2.14) gives

$$
E(t) \leq J(t) \leq J\left(t_{0}^{\prime}\right) e^{-4\left(t-t_{0}^{\prime}\right)+\beta} \leq 5 c_{4} E e^{-4\left(t-t_{0}^{\prime}\right)} .
$$

Finally, we convert this energy bound to a pointwise bound by applying (2.6). This yields the bound of Proposition 2.1a.

Case (b) When $J\left(t_{0}\right) \leq 0$, we set $E(t)=E\left(t_{0}, t\right)$. This time (2.5) and (2.9) give

$$
0 \leq E(t)=J\left(t_{0}\right)-J(t) \leq-J(t) \leq c_{4} E \leq c_{4} \varepsilon_{0}
$$

for all $t \in\left[t_{0}, t_{1}\right]$, and $J^{\prime}=-E^{\prime} \leq 0$ is again given by (2.15). Note that $J(t) \leq 0$ now. The analysis following (2.15) still applies, giving (2.16) with $J^{\prime}=-|J|^{\prime}$. Again we impose (2.17) and integrate twice. First, (2.16) and (2.17) give $|J|^{\prime} \geq 2|J|$, and hence $|J(t)| \leq\left|J\left(t_{1}^{\prime}\right)\right| e^{2\left(t-t_{1}^{\prime}\right)}$ with $\left|J\left(t_{1}^{\prime}\right)\right| \leq$ $\left(8 c_{7}\right)^{-2}$ by (2.19) and (2.17). Putting this back into (2.16) (with $J^{\prime}$ replaced by $\left.-|J|^{\prime}\right)$ and integrating from $t$ to $t_{1}^{\prime}$ gives $\log \left|J\left(t_{1}^{\prime}\right)\right|-\log |J(t)| \geq 4\left(t_{1}^{\prime}-\right.$ $t)-3 / 2$, and hence

$$
E(t) \leq|J(t)| \leq 8\left|J\left(t_{1}^{\prime}\right)\right| e^{-4\left(t_{1}^{\prime}-t\right)} \leq 5 c_{4} E e^{-4\left(t_{1}^{\prime}-t\right)}
$$

using (2.19). Again, (2.6) converts this to a pointwise bound, yielding the bound of Proposition 2.1b.

Looking over the above proof one sees that the constants $T, C, \varepsilon_{0}$ depend only on the constant $C(g)$ used in the definition (1.9) of the asymptotically cylindrical metric. This completes the proof of Proposition 2.1.

As noted after equation (2.9) Theorem 1.3ab follows immediately from Proposition 2.1.

Theorem 1.1ab now follows from Theorem 1.3 by applying a conformal transformation. Specifically, using normal coordinates $\left\{x^{i}\right\}$ on $B=B(p, \rho)$ and coordinates $\left(t,\left\{y^{i}\right\}\right)$ on $\mathbb{R} \times S^{3} \subset \mathbb{R} \times \mathbb{R}^{4}$, define a diffeomorphism $\phi:[T, \infty] \times S^{3} \rightarrow B-\{0\}$ by $x^{i}=\phi^{i}(t, y)=\rho e^{T-t} y^{i}$. Then $r=|x|=$ $\rho e^{T-t}$. The metric on $B$ has a normal coordinate expansion $g_{i j}=\delta_{i j}+$ $h_{i j k l} x^{k} x^{l}$ where $h$ is bounded in $C^{2}$, uniformly in $p \in M$ since $M$ has bounded geometry. Noting that $d x^{i}=r\left(d y^{i}-y^{i} d t\right)$ and that $\sum y^{i} d y^{i}=0$ (since $\sum y^{i} y^{i}=1$ on $\mathbb{R} \times S^{3}$ ) we find that

$$
\phi^{*}\left(r^{-2} g\right)=\phi^{*}\left(r^{-2} g_{i j} d x^{i} d x^{j}\right)=\sum\left(d y^{i}\right)^{2}+d t^{2}+e^{-2 t} R
$$


where $R$ is bounded in $C^{2}$. Thus $g^{\prime}=\phi^{*}\left(r^{-2} g\right)$ is an asymptotically cylindrical metric on $\mathbb{R} \times S^{3}$.

Now suppose that $A$ is a SD (resp. ASD) connection on $B$ as in Theorem 1.1. Then $A$ is also SD (resp. ASD) with respect to the metric $r^{-2} g$ on $B-$ $\{0\}$ and satisfies $\|F\|_{B, r^{-2} g} \leq \varepsilon_{0}$ since the Yang-Mills energy is conformally invariant. Since $\phi$ reverses orientation, $\phi^{*} A$ is ASD (resp. SD) on $[T, \infty] \times S^{3}$, and for $t \geq T$ the energy of $\phi^{*} A$ on $[t, \infty] \times S^{3}$ is the energy $E(B(p, r))$ of $A$ where $r=\rho e^{T-t}$. Thus if $A$ satisfies the hypotheses of Theorem 1.1a then $\phi^{*} A$ satisfies the hypotheses of Theorem 1.3a with $t_{0}=T+\log \left(\rho / r_{1}\right)$ and $t_{1}=T+\log \left(\rho / r_{0}\right)$. Therefore $\phi^{*} F$ satisfies (1.11) for the metric $g^{\prime}$. In fact, a slight modification of the proof of Theorem 1.3 shows that (1.11) holds (with a different constant) on the larger region $\left[t_{0}+\frac{1}{2}, t_{1}-\frac{1}{2}\right] \times S^{3}$, which corresponds under $\phi$ to an annulus containing $\Omega\left(p, 2 r_{0}, r_{1} / 2\right)$. The dependence of $|F|$ on the metric is clear from the local expression $|F|^{2}=$ $-\operatorname{tr}\left(g^{i j} g^{k l} F_{i k} F_{j l}\right)$, so for $y \in \Omega\left(p, 2 r_{0}, r_{1} / 2\right)$ (1.11) translates into

$\left|F_{A}(y)\right|_{g}=r^{-2}\left|\phi^{*} F(t, y)\right|_{g^{\prime}} \leq C r^{-2} e^{-2\left(t-t_{0}\right)}\left\|\phi^{*} F\right\|_{\left[t_{0}, t_{1}\right] \times S^{3}}=\frac{C}{r_{1}^{2}}\|F\|_{\Omega}$,

which is (1.3). Similarly; (1.12) translates into (1.4).

The proof that Theorem 1.3ab implies Theorem 1.2ab is completely parallel, this time using the diffeomorphism $\phi:[T, \infty] \rightarrow \Omega(R, \infty)$ by $\phi\left(t, y^{i}\right)=R e^{t-T} y^{i}$ and the metric $g^{\prime}=\phi^{*}\left(r^{-2} g\right)$. The details are left to the reader.

\section{General Yang-Mills Fields.}

We now consider Yang-Mills fields that are not necessarily SI) or ASD and establish the pointwise bounds (1.2), (1.6), and (1.10). For the main part of the argument we work on an asymptotically flat manifold, proving (1.6) by adapting the technique of Røade [R]. The inequalities (1.2) and (1.10) then follow by conformal transformations.

The key to the proof is to use the "Kato-Yau" inequality to obtain a sharp pointwise differential inequality for $|F|$. The Kato-Yau inequality is a strengthened version of the inequality $|d| F|| \leq\left|\nabla_{A} F\right|$ that is usually employed in this context (cf. [U]); the strengthening is possible because some of the components of $\nabla F$ vanish by the Yang-Mills equations $d_{A} F=$ $d_{A}^{*} F=0$. For a simple proof see $[\mathrm{R}]$. 
Lemma 3.1 (Kato-Yau inequality). If $A$ is a Yang-Mills connection and $F=F_{A}$ then

$$
\left.|d| F\right|^{2} \leq \frac{2}{3}\left|\nabla_{A} F\right|^{2}
$$

The Yang-Mills equations also mean that $\Delta_{A} F=\left(d_{A} d_{A}^{*}+d_{A}^{*} d_{A}\right) F$ vanishes, so by the Weitzenböck formula

$$
0=\Delta F=\nabla_{A}^{*} \nabla_{A} F+\mathcal{R} F+[F, F]
$$

where $\nabla$ is the covariant derivative of the connection $A$, and $\mathcal{R}=s / 3+W$ is an endomorphism constructed from the scalar curvature $s$ and the Weyl curvature $W$. Using (3.1) and (3.2), $u=|F|^{1 / 2}$ satisfies

$$
\begin{aligned}
\Delta u & =\frac{1}{4} u^{-3}\left[2\left\langle F, \nabla_{A}^{*} \nabla_{A} F\right\rangle-2|\nabla F|^{2}+\left.3|d| F\right|^{2}\right] \\
& \leq \frac{1}{2} u^{-3}\langle F, \mathcal{R} F+[F, F]\rangle
\end{aligned}
$$

so

$$
\Delta u \leq|\mathcal{R}| u+c_{1} u^{3} .
$$

As in Theorem 1.2 we fix an asymptotically flat manifold $(M, g)$ and an annulus $\Omega\left(r_{0}, r_{1}\right)$ in the asymptotic end. We will prove the bound (1.2) by repeatedly integrating (3.3).

First, if the energy is sufficiently small, Uhlenbeck's pointwise bound gives

$$
u \leq \frac{\sqrt{c_{2} \varepsilon}}{r} \text { on } \Omega\left(2 r_{0}, r_{1} / 2\right) \quad \text { where } \quad \varepsilon=\|F\|_{\Omega\left(r_{0}, r_{1}\right)}
$$

([U] Theorem 3.5). Putting this into (3.3) and noting that $|\mathcal{R}| \leq c_{3} r^{-4}$ by the asymptotically flat condition, we have

$$
\Delta u \leq\left(c_{3} r^{-4}+C E r^{-2}\right) u \text { on } \Omega\left(2 r_{0}, r_{1} / 2\right) .
$$

The second ingredient is the following comparison principle, which is essentially due to J. Røade [R]. 
Lemma 3.2. Let $h$ be a positive harmonic function on a domain $\Omega$ in a Riemannian manifold. Set $\xi=|d(\log \sqrt{h})|^{2}$. Then for any $a \geq-1$ the operator $L=\Delta+a \xi$ satisfies the comparison principle on $\Omega$. Specifically, if $u, w \in L^{1,2}(\Omega)$ with $u \leq w$ weakly on $\partial \Omega$ and $L u \leq L w$ weakly on $\Omega$, then $u \leq w$ a.e. on $\Omega$.

Proof. If $\phi$ vanishes on $\partial \Omega$ then, squaring and integrating by parts,

$$
0 \leq \int_{\Omega}\left|d \phi-\frac{1}{2} \phi d(\log h)\right|^{2} d v=\int_{\Omega}|d \phi|^{2}-\frac{1}{2} \phi^{2} d^{*} d(\log h)+\xi \phi^{2} d v .
$$

But $d^{*} d(\log h)=d^{*}\left(h^{-1} d h\right)=h^{-2}|d h|^{2}=4 \xi$ since $d^{*} d h=0$. Thus

$$
0 \leq \int_{\Omega}|d \phi|^{2}-\xi \phi^{2} d v
$$

with equality if and only if $\phi=0$. Now take $\phi=u-w$. By hypothesis $\phi_{+}=\max \{0, \phi\}$ vanishes on $\partial \Omega$ and $L \phi \leq 0$ weakly on $\Omega$. Hence

$$
0 \geq \int_{\Omega}\left\langle\phi_{+}, L \phi\right\rangle d v=\int_{\Omega}\left\langle d \phi_{+}, d \phi\right\rangle+a \xi \phi_{+} \phi d v \geq \int_{\Omega}\left|d \phi_{+}\right|^{2}-\xi \phi_{+}^{2} d v .
$$

Therefore $\phi_{+}=0$. The lemma follows.

To apply Lemma 3.2, we need a positive harmonic function $h$ where both $h$ and $\xi=|d(\log \sqrt{h})|^{2}$ are essentially $1 / r^{2}$ near infinity. The existence of such a function is ensured by the following proposition, which is proved in the appendix.

Proposition 3.3. Let $(M, g)$ be an asymptotically flat four-manifold. Then there is an $R_{0}$ such that for each $R>R_{0}$ there is a positive harmonic function $h$ on $D=M_{\infty}-B(R)$ with $h=1 / R^{2}$ on $\partial D$,

$$
\frac{3}{4 r^{2}} \leq h \leq \frac{4}{3 r^{2}} \quad \text { and } \quad h \leq 8 \xi \text {. }
$$

Now fix an annulus $\Omega=\Omega\left(2 r_{0}, r_{1} / 2\right)$ with $4 R_{0} \leq 4 r_{0} \leq r_{1}$ where $R_{0} \geq 1$ is large enough that Proposition 3.3 applies. Suppose that $A$ is a Yang-Mills connection on $\Omega$ with

$$
\varepsilon=\|F\|_{\Omega} \leq \varepsilon_{0}=1 / 16 C .
$$


Then using (3.4), (3.5), (3.7), and (3.8)

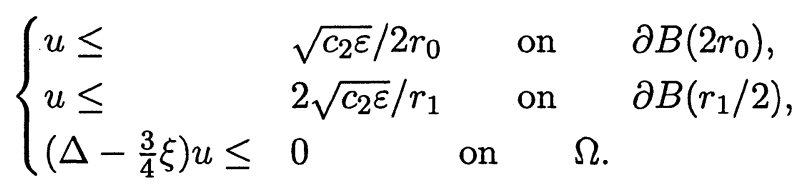

On the other hand,

$$
\Delta h^{\alpha}=d^{*} d h^{\alpha}=\alpha(1-\alpha) h^{\alpha}\left|h^{-1} d h\right|^{2}=4 \alpha(1-\alpha) \xi h^{\alpha},
$$

so $\left(\Delta-\frac{3}{4} \xi\right) h^{\alpha}=-4(\alpha-3 / 4)(\alpha-1 / 4) \xi h^{\alpha}$. Consequently, the function

$$
w=\sqrt{2 C E}\left[\sqrt{r_{0}}\left(\frac{4}{3} h\right)^{3 / 4}+\frac{1}{\sqrt{r_{1}}}\left(\frac{4}{3} h\right)^{1 / 4}\right]
$$

satisfies

$$
\left\{\begin{array}{llll}
w \geq & \sqrt{c_{2} \varepsilon} / 2 r_{0} & \text { on } & \partial B\left(2 r_{0}\right) \\
w \geq & 2 \sqrt{c_{2} \varepsilon} / r_{1} & \text { on } & \partial B\left(r_{1} / 2\right), \\
\left(\Delta-\frac{3}{4} \xi\right) w= & 0 . & &
\end{array}\right.
$$

Applying Lemma 3.2, we conclude that $u \leq w$ on $\Omega$.

Returning to the differential inequality (3.3) we now have $\Delta u \leq|\mathcal{R}| w+$ $c_{1} w^{3} \leq \sqrt{2 C E} \psi$, where, using (3.8) and noting that $|\mathcal{R}| \leq c_{3} r^{-4} \leq c_{4} h^{2}$ and $h^{\alpha} \leq 8 \xi h^{\alpha-1}$ by (3.7),

$$
\psi=\xi\left\{11 c_{4} \sqrt{r_{0}} h^{7 / 4}+10\left(c_{4} r_{1}^{-1 / 2}+c_{1} r_{0}^{3 / 2}\right) h^{5 / 4}+3 c_{1} r_{1}^{-3 / 2} h^{-1 / 4}\right\} .
$$

Then the function

$$
H=r_{0} h+\frac{1}{r_{1}}
$$

is harmonic, and by (3.10)

$$
\eta=\frac{44}{21} c_{4} \sqrt{r_{0}} h^{7 / 4}+8\left(c_{4} r_{1}^{-1 / 2}+c_{1} r_{0}^{3 / 2}\right) h^{5 / 4}+\frac{12}{5} c_{1} r_{1}^{-3 / 2} h^{-1 / 4}
$$

satisfies $\Delta \eta=-\psi$. Moreover, one checks that there is a constant $c_{5}$ such that $|\eta| \leq c_{5} H$ on $\Omega$. Set $c_{6}=c_{5}+\sqrt{2}$. Then

$$
\phi=\sqrt{2 C E}\left(c_{6} H-\eta\right) .
$$


satisfies $\phi \geq \sqrt{2} H$ and, using (3.9),

$$
\left\{\begin{array}{llll}
u-\phi \leq & 0 & \text { on } & \partial \Omega \\
\Delta(u-\phi)= & 0 & \text { on } & \Omega
\end{array}\right.
$$

The maximum principle for the Laplacian then gives $u \leq \phi \leq 2 c_{i j} \sqrt{c_{2} \varepsilon} H$, so with (3.7) we have

$$
|F|=u^{2} \leq 2 c_{6}^{2} C E\left(r_{0} h+\frac{1}{r_{1}}\right)^{2} \leq c E\left(\frac{r_{0}^{2}}{r^{4}}+\frac{1}{r_{1}^{2}}\right) .
$$

This establishes the bound (1.6) of Theorem 1.2. The corresponding bounds (1.2) and (1.10) follow easily by applying conformal transformations, just as we did at the end of section 2 .

\section{Applications to concentrated instantons.}

We now apply Theorems $1.1-1.3$ to obtain pointwise bounds on the curvature of SD/ASD connections near the boundary of moduli space. Such connections consist of one or more sharply concentrated "instantons" superimposed on a smooth background connection. We begin this section by giving a precise definition of concentrated connections and showing that such connections indeed form a neighborhood of the boundary of moduli space. We then use the results of section 1 to obtain pointwise bounds on the curvature $|F|$ of concentrated connections. In fact we obtain separate bounds for the regions close to the instantons (Proposition 4.4), for the intermediate collars around the instantons (Lemma 4.3d), and for the regions far from the instantons (Proposition 4.6).

Throughout this section we will consider SD or ASD connections on a fixed unitary bundle over a compact Riemannian manifold $(M, g)$. The energy density $f=f_{A}=\frac{1}{8 \pi^{2}}\left|F_{A}\right|^{2} d v_{g}$ then satisfies

$$
\int_{M} f=k
$$

for some integer $k \geq 0$. Let $\rho$ and $\varepsilon_{0}$ be as in Theorem 1.1 and set

$$
\delta_{0}=\min \left\{\frac{1}{16}, \frac{\varepsilon_{0}}{2 k}\right\} \text {. }
$$

Definition 4.1. A SD/ASD connection $A$ is $\varepsilon$-concentrated if there exist positive integers $k_{i}$, points $p_{i} \in M$, and numbers $\varepsilon_{i}, \rho_{i}>0$, with $\varepsilon_{i} \leq \varepsilon$ and $32 \varepsilon_{i} \leq \rho_{i} \leq \rho$ such that the balls $B\left(p_{i}, \rho_{i}\right)$ are disjoint and for each $i$ 
(a)

$$
\int_{B\left(p_{i}, \varepsilon_{i}\right)} f=k_{i}-\delta_{0}, \quad \text { and }
$$

(b)

$$
\int_{B\left(p_{i}, \rho_{i}\right)} f<k_{i} .
$$

We will refer to the collection $\left\{\left(p_{i}, \varepsilon_{i}, \rho_{i}, k_{i}\right)\right\}$ as concentration data for $A$. In general concentration data are not unique.

Thus an $\varepsilon$-concentrated connection has "core regions" $B\left(p_{i}, \varepsilon_{i}\right)$ with nearly integral energy $k_{i}>0$ surrounded and isolated by low-energy annuli $B\left(p_{i}, \rho_{i}\right)-B\left(p_{i}, \varepsilon_{i}\right)$ of modulus $\rho_{i} / \varepsilon_{i} \geq 32$.

Remarks. (1) For many purposes it suffices to take $\rho_{i}=32 \varepsilon_{i}$, thereby simplifying Definition 4.1. However, by including $\rho_{i}$ as independent parameters we will obtain bounds that get better as the moduli $\rho_{i} / \varepsilon_{i}$ increase.

(2) Some of the estimates below are more easily proved if one assumes that the points $p_{i}$ are separated by a fixed distance. We avoid making this assumption in order to get bounds that hold uniformly for families of concentrated connections with two or more of the points $p_{i}$ coalescing.

The importance of Definition 4.1 is that the $\varepsilon$-concentrated connections are a neighborhood of the boundary of moduli space. This statement is made precise in the next proposition. Let $\mathcal{M}$ denote the SD or ASD moduli space (on our fixed degree $k$ unitary bundle) and let $\mathcal{M}^{\varepsilon} \subset \mathcal{M}$ denote the subset of $\varepsilon$-concentrated connections. As is standard, we topologize $\mathcal{M}$ with the $L_{3}^{2}$ Sobolev topology.

Proposition 4.2. $\mathcal{M}-\mathcal{M}^{\varepsilon}$ is compact for each $\varepsilon \leq \rho / 32$.

Proof. Given a sequence $\left\{\left[A_{n}\right]\right\}$ in $\mathcal{M}$, Uhlenbeck's Compactness Theorem says there is a subsequence that either converges, or whose energy densities $f_{n}$ converge as measures to a smooth non-negative measure $f_{0}$ plus a nonempty set of point masses at points $p_{i} \in M$ :

$$
f_{n} \rightarrow f_{0}+\sum k_{i} \delta_{p_{i}}
$$


where $k_{i} \geq 1$ and $k_{0}=\int_{M} f_{0}$ are integers ([DK] $\left.\S 4.4\right)$. We can therefore choose small numbers $\varepsilon_{i}^{\prime}, 0<\varepsilon_{i}^{\prime} \leq \varepsilon$, set $\rho_{i}=32 \varepsilon_{i}^{\prime}$, and then choose a large number $N$ such that such that the balls $B\left(p_{i}, \rho_{i}\right)$ are disjoint and

$0 \leq \int_{B\left(p_{i}, \rho_{i}\right)} f_{0}<\delta_{0} / 2, \quad$ and $\quad\left|\int_{B\left(p_{i}, \rho_{i}\right)} f_{n}-f_{0}-\sum k_{i} \delta_{p_{i}}\right|<\delta_{0} / 2$ for each $i$ and $n \geq N$. Then

$$
\int_{B\left(p_{i}, \varepsilon_{i}^{\prime}\right)} f_{n} \geq \int_{B\left(p_{i}, \varepsilon_{i}^{\prime}\right)}\left(f_{0}+\sum k_{i} \delta_{p_{i}}\right)-\delta_{0} / 2 \geq k_{i}-\delta_{0} / 2 .
$$

Hence for each $n \geq N$ the numbers

$$
\varepsilon_{i}^{n}=\inf \left\{\varepsilon>0 \mid \int_{B\left(p_{i}, \rho_{i}\right)} f_{n} \geq k_{i}-\delta_{0}\right\}
$$

satisfy $\varepsilon_{i}^{n} \leq \varepsilon_{i}^{\prime}$ and $\int_{B\left(p_{i}, \varepsilon_{i}^{n}\right)} f_{n}=k_{i}-\delta_{0}$. Furthermore, $32 \varepsilon_{i}^{n} \leq \rho_{i}$ and by (4.3)

$$
\int_{B\left(p_{i}, \rho_{i}\right)} f_{n} \leq \int_{B\left(p_{i}, \varepsilon_{i}^{n}\right)} f_{n}+\int_{B\left(p_{i}, \rho_{i}\right)-B\left(p_{i}, \varepsilon_{i}^{n}\right)} f_{n} \leq\left(k_{i}-\delta_{0}\right)+\delta_{0}=k_{i} .
$$

Thus $\left\{\left[A_{n}\right]\right\}$ has a subsequence that either converges or eventually enters $\mathcal{M}^{\varepsilon}$. The proposition follows since $\mathcal{M}^{\varepsilon}$ is open.

Now suppose that $A$ is an $\varepsilon$-concentrated connection, so the properties of Definition 4.1 hold for some data $\left\{p_{i}, \varepsilon_{i}, \rho_{i}\right\}$. Fixing $\left\{p_{i}, \varepsilon_{i}, \rho_{i}\right\}$, we define center points $\bar{p}_{i} \in M$ of $A$ (one for each $i$ ) to be the points with coordinates

$$
\bar{p}_{i}^{l}=\frac{\int_{B\left(p_{i}, 4 \varepsilon_{i}\right)} x^{l} f}{\int_{B\left(p_{i}, 4 \varepsilon_{i}\right)} f}
$$

in normal coordinates $\left\{x^{l}\right\}$ on $B\left(p_{i}, 4 \varepsilon_{i}\right)$. We then define the scales $\lambda_{i}>0$ of $A$ by

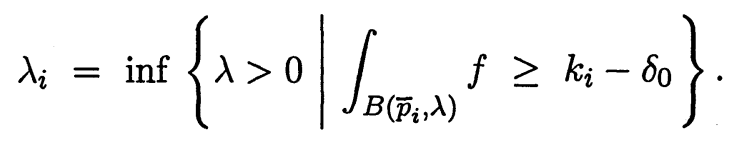

Neither the center points nor the scales are canonically defined for two reasons. First, one can replace $\left\{p_{i}, \varepsilon_{i}, \rho_{i}\right\}$ by different data $\left\{p_{i}^{\prime}, \varepsilon_{i}^{\prime}, \rho_{i}^{\prime}\right\}$ for 
which the properties of Definition 4.1 hold, obtaining different centers and scales. The bounds we will obtain in this section hold with uniform constants for any choice of data $\left\{p_{i}, \varepsilon_{i}, \rho_{i}\right\}$. Second, the constants ' $4 \varepsilon_{i}$ ' in (4.4) and ' $\delta_{0}$ ' in (4.5) are a convention that can be changed, for example, one could integrate over $B\left(p_{i}, 8 \varepsilon_{i}\right)$ in (4.4), or replace the quantity $k_{i}-\delta_{0}$ in (4.5) by $k_{i}-\frac{1}{2}$. The bounds below hold (after adjusting the constants) for such variations on definitions (4.4) and (4.5). In particular, in section 5 we will show that they hold for the specific choices of center and scale used in [D], [GP2], and [G1,G2].

The next lemma states some simple consequences of definitions 4.1, 4.4, and 4.5, and of Theorem 1.1. Write $E(D)$ for the integral of $f=f_{A}$ over a domain $D$, and $\Omega(p, a, b)$ for the annulus $\overline{B(p, b)-B(p, a)}$.

Lemma 4.3. If $A$ is $\varepsilon$-concentrated then

(a) there are at most $\frac{16}{15} k$ points $p_{i}$,

(b) the energy $E_{0}$ on $M-\cup B\left(p_{i}, \varepsilon_{i}\right)$ satisfies $E_{0} \leq k_{0}+\varepsilon_{0}$ where $k_{0}=$ $k-\sum k_{i}$

(c) $\operatorname{dist}\left(p_{i}, \bar{p}_{i}\right) \leq \frac{4}{3} \varepsilon_{i} \leq \frac{1}{24} \rho_{i}$ and $\lambda_{i}<\frac{7}{3} \varepsilon_{i}<\frac{1}{12} \rho_{i}$,

(d) writing $r_{i}=\operatorname{dist}\left(\bar{p}_{i}, \cdot\right)$, there is a constant $c_{1}$ such that

$$
\left|F_{A}\right|=\left|8 \pi^{2} f_{A}\right|^{1 / 2} \leq c_{1} \frac{\lambda_{i}^{2}}{r_{i}^{4}} \sqrt{\delta_{0}} \quad \text { on } \quad \Omega\left(\bar{p}_{i}, 2 \lambda_{i}, \frac{4}{5} \rho_{i}\right),
$$

Proof. (a) Since $k_{i}-\delta \geq \frac{1}{16}$ equation (4.1) gives $k \geq \sum\left(k_{i}-\delta_{0}\right) \geq \sum \frac{15}{16}$.

(b) From (4.1) we also have $k \geq E_{0}+\sum\left(k_{i}-\delta_{0}\right)$ with $\sum \delta_{0} \leq \frac{16}{15} k \delta_{0} \leq \varepsilon_{0}$.

(c) By (4.1), (4.2), and (4.4) the denominator of (4.4) is at least $k_{i}-\delta_{0}$, and hence

$$
\begin{aligned}
\operatorname{dist}\left(p_{i}, \bar{p}_{i}\right) & \leq \frac{1}{k_{i}-\delta_{0}}\left[\varepsilon_{i} \int_{B\left(p_{i}, \varepsilon_{i}\right)} f+4 \varepsilon_{i} \int_{\Omega\left(p_{i}, \varepsilon_{i}, 4 \varepsilon_{i}\right)} f\right] \\
& \leq \frac{\varepsilon_{i}}{k_{i}-\delta_{0}}\left[k_{i}+4 \delta_{0}\right] \leq \frac{4}{3} \varepsilon_{i} .
\end{aligned}
$$

Then $E\left(B\left(\bar{p}_{i}, \frac{7}{3} \varepsilon_{i}\right)\right) \geq E\left(B\left(p_{i}, \varepsilon_{i}\right)\right)=k_{i}-\delta_{0}$, so $\lambda_{i} \leq \frac{7}{3} \varepsilon_{i}$. Finally, $32 \varepsilon_{i} \leq \rho_{i}$ by definition 4.1 . 
(d) Using (c) we have $k_{i}-1 / 2 \leq k_{i}-\delta_{0} \leq E\left(B\left(\bar{p}_{i}, \frac{23}{24} \rho_{i}\right)\right) \leq$ $E\left(B\left(p_{i}, \rho_{i}\right)\right)<k_{i}$ and $E\left(\Omega\left(\bar{p}_{i}, \lambda_{i}, \frac{23}{24} \rho_{i}\right)\right) \leq E\left(B\left(p_{i}, \rho_{i}\right)\right)-E\left(B\left(\bar{p}_{i}, \lambda_{i}\right)\right)<$ $k_{i}-\left(k_{i}-\delta_{0}\right)=\delta_{0}<\varepsilon_{0}$. Hence Theorem 1.1b applies on $\Omega\left(c, \lambda_{i}, \frac{23}{24} \rho_{i}\right)$, giving (4.6).

Our goal now is to obtain pointwise bounds on $\left|F_{A}\right|$ for $\varepsilon$-concentrated $\mathrm{SD} / \mathrm{ASD}$ connections. Lemma 4.3d gives such a bound in the annular regions $\Omega\left(\bar{p}_{i}, 2 \lambda_{i}, 4 \rho_{i} / 5\right)$. In general there is no universal bound of this form inside the core regions $B\left(\bar{p}_{i}, \lambda_{i}\right)$ because there may be "bubbles-on-bubbles". For example, one can have a 1-parameter family of $k=2$ connections $A_{t}$ on $B(p, \lambda)$ that split into two $k=1$ instantons with scales $t \lambda$ where $t \rightarrow 0$; for such a family there is no uniform pointwise bound on $|F|$. However, this phenomenon cannot occur in any $B\left(\bar{p}_{i}, \lambda_{i}\right)$ with $k_{i}=1$, and in that case a renormalization argument, essentially due to Uhlenbeck, yields the following pointwise bound. Because our definitions of scale and center are not completely standard, we give the argument in its entirety.

Proposition 4.4. There are constants $c_{2}, \varepsilon_{1}>0$ such that if $A$ is any $\varepsilon_{1}$ concentrated unitary SD/ASD connection then for each $i$ with $k_{i}=1$ we have

$$
\left|F_{A}\right| \leq \frac{c_{2}}{\lambda_{i}^{2}} \quad \text { on } \quad B\left(\bar{p}_{i}, 2 \lambda_{i}\right)
$$

Proof. Suppose not. Then there is a sequence $\left\{A_{n}\right\}$ of $\frac{1}{n}$-concentrated SD/ASD connections and balls $B\left(p_{n}, 32 / n\right)$ on which the energy density $f_{n}$ of $A_{n}$ satisfies (4.1) and (4.2) with $\varepsilon_{n}=1 / n, \rho_{n}=32 / n$, and $k_{i}=1$, and there are points $z_{n} \in B\left(\bar{p}_{n}, 2 \lambda_{n}\right)$ where the curvature $F_{n}$ of $A_{n}$ satisfies

$$
\left|F_{n}\left(z_{n}\right)\right| \geq n \lambda_{n}^{-2}
$$

(Here we are suppressing a subscript: $p_{n}=p_{n, i}$ etc.) Let $w_{n}$ be a point in $B\left(\bar{p}_{n}, 2 \lambda_{n}\right)$ at which $\left|F_{n}\right|$ attains its supremum. Using normal coordinates centered at $p_{n}$ we can regard the $A_{n}$ as connections on $B(0,32 / n) \subset \mathbb{R}^{4}$, and pullback by the transformation $\phi_{n}(x)=L_{n}\left(x-w_{n}\right)$ where

$$
L_{n}^{2}=\left|F_{n}\left(w_{n}\right)\right|=\sup _{B\left(\bar{p}_{n}, 2 \lambda_{n}\right)}\left|F_{n}\right| .
$$

Since $B\left(w_{n}, \lambda_{n}\right) \subset B\left(\bar{p}_{n}, 3 \lambda_{n}\right) \subset B\left(p_{n}, 32 / n\right)$ by Lemma $4.3 c$ the connections $A_{n}^{\prime}=\phi_{n}^{*} A_{n}$ are SD/ASD with respect to metrics $g^{\prime}=\phi_{n}^{*} g$ that converge to the euclidean metric uniformly in $C^{2}$ on compact sets. In fact, by 
(4.8) and (4.9) these balls have radii

$$
L_{n} \lambda_{n} \geq \sqrt{n} \rightarrow \infty \text {. }
$$

Furthermore, for each $y \in B\left(0, L_{n} \lambda_{n}\right)$ the curvature $F_{n}^{\prime}$ of $A_{n}^{\prime}$ satisfies $\left|F_{n}^{\prime}(y)\right| \leq L_{n}^{-1}\left|F_{n}\left(\phi_{n}^{-1}(y)\right)\right| \leq 1$ and $\left|F_{n}^{\prime}(0)\right|=1$, while by (4.2) (with $k_{i}=1$ ) the energy density $f_{n}^{\prime}$ of $A_{n}^{\prime}$ satisfies

$$
\int_{B(0, \sqrt{n})} f_{n}^{\prime} \leq \int_{B\left(w_{n}, \lambda_{n}\right)} f_{n} \leq 1 .
$$

By the Uhlenbeck Compactness Theorem ([FU]) a subsequence of the connections $\left\{A_{n}^{\prime}\right\}$ converges uniformly in $C^{1}$ on compact sets to a SD/ASD $k=1$ connection $A_{\infty}^{\prime}$ on $R^{4}$ with the euclidean metric $\left(A_{\infty}^{\prime}\right.$ cannot have $k=0$ since $\left.\left|F_{n}^{\prime}(0)\right|=1\right)$. Hence there are numbers $L, N>0$, depending on $\delta_{0}$, such that

$$
\int_{B(0, L)} f_{n}^{\prime} \geq 1-\delta_{0} \quad \forall n \geq N
$$

But $B(0, L)$ corresponds to $B\left(w_{n}, L / L_{n}\right)$ in the original coordinates, so using (4.10)

$$
\int_{B\left(w_{n}, \frac{L}{\sqrt{n}} \lambda_{n}\right)} f_{n} \geq 1-\delta_{0} \quad \forall n \geq N
$$

Thus for large $n$ most of the energy is concentrated in a ball around $w_{n}$ whose radius is much smaller than $\lambda_{n}$. To complete the proof we will show that this implies that the center $\bar{p}_{n}$ lies close enough to $w_{n}$ that (4.11) contradicts the definition (4.5) of $\lambda_{n}$.

Set $\mu_{n}=L \lambda_{n} / \sqrt{n}$. By (4.5) and (4.1) we have, in normal coordinates on $B\left(p_{n}, 32 / n\right)$,

$\left|\left(\bar{p}_{n}-w_{n}\right)^{l}\right|=\frac{\int_{B\left(p_{n}, 4 / n\right)}\left(x-w_{n}\right)^{l} f_{n}}{\int_{B\left(p_{n}, 4 / n\right)} f_{n}} \leq\left(1-\delta_{0}\right)^{-1} \int_{B\left(p_{n}, 4 / n\right)}\left(x-w_{n}\right)^{l} f_{n}$.

Note that $\operatorname{dist}\left(p_{n}, w_{n}\right) \leq \operatorname{dist}\left(p_{n}, \bar{p}_{n}\right)+2 \lambda_{n} \leq 6 / n$ by Lemma $4.3 \mathrm{c}$, so $B\left(p, 4 \varepsilon_{n}\right) \subset B(w, 10 / n) \subset B(w, 20 / n) \subset B\left(p_{n}, 32 / n\right)$. As in the proof of Lemma 4.3d these inclusions imply that $1 / 2<E\left(B\left(w_{n}, 20 / n\right)\right)<1$ and $E\left(\Omega\left(w_{n}, \mu_{n}, 20 / n\right)\right) \leq \varepsilon_{0}$, so Theorem $1.1 \mathrm{~b}$ gives

$$
\left|F_{n}\right| \leq c_{1} \frac{\mu_{n}^{2}}{\operatorname{dist}^{4}\left(w_{n}, \cdot\right)} \sqrt{\delta_{0}} \quad \text { on } \Omega\left(w_{n}, 2 \mu_{n}, 10 / n\right) .
$$


Using this and integrating in polar coordinates on $B\left(p_{n}, 32 / n\right)$, noting that the metric is uniformly equivalent to the euclidean metric,

$$
\begin{aligned}
\frac{1}{2}\left(1-\delta_{0}\right) \operatorname{dist}\left(\bar{p}_{n}, w_{n}\right) & \leq \int_{B\left(p_{n}, 2 \mu_{n}\right)}\left|x-w_{n}\right| f_{n}+\int_{B\left(w_{n}, 10 / n\right)}\left|x-w_{n}\right| f_{n} \\
& \leq 2 \mu_{n} \int_{B\left(p_{n}, 2 \mu_{n}\right)} f_{n}+c_{3} \int_{2 \mu_{n}}^{10 / n} r \cdot c_{1}^{2} \delta_{0} \frac{\mu_{n 2}^{4}}{r^{8}} \cdot r^{3} d r .
\end{aligned}
$$

Bounding the first integral by (4.2) (we have $B\left(w_{n}, 2 \mu_{n}\right) \subset B\left(p_{n}, 32 / n\right)$ for large $n$ ) and integrating the second gives

$$
\operatorname{dist}\left(\bar{p}_{n}, w_{n}\right) \leq \frac{32}{15}\left[2 \mu_{n}+c_{4} \delta_{0} \mu_{n}\right] \leq c_{5} \mu_{n}
$$

Hence by (4.11)

$$
\int_{B\left(\bar{p}_{n},\left(1+c_{5}\right) \mu_{n}\right)} f_{n} \geq \int_{B\left(w_{n}, \mu_{n}\right)} f_{n} \geq 1-\delta_{0} .
$$

Comparing this with the definition (4.5) of $\lambda_{n}$ and recalling the definition of $\mu_{n}$

$$
\lambda_{n} \leq\left(1+c_{5}\right) \mu_{n}=\frac{4\left(1+c_{5}\right) L}{\sqrt{n}} \lambda_{n} .
$$

Taking $n$ sufficiently large gives the desired contradiction.

Lemma 4.3d and Proposition 4.4 provide local pointwise bouncls for $|F|$ near instantons. To obtain global pointwise bounds we also need estimates in the region outside the balls surrounding the instantons. By Lemma 4.3b this region has energy $E_{0} \leq k_{0}+\varepsilon_{0}$, where $k_{0}=k-\sum k_{i}$ is the energy of a (non-canonical) background SD/ASD connection on $M$. We first consider the case $k_{0}=0$.

Proposition 4.5. There are constants $C, \varepsilon_{2}>0$ such that if $A$ is any $\varepsilon_{2}$ concentrated $S D / A S D$ connection with concentration data for whici $k_{0}=0$, we have

$$
\left|F_{A}(y)\right| \leq \frac{C \sqrt{\delta_{0}}}{r^{2}} \sum_{i} \frac{\lambda_{i}^{2}}{\rho_{i}^{2}} \quad \forall y \in M-\cup B\left(\bar{p}_{i}, \rho_{i} / 2\right) .
$$

where $r_{i}=\operatorname{dist}\left(\bar{p}_{i}, \cdot\right)$ and $r=\min _{i} r_{i}$. 
Proof. Fix $y \in M-\cup B\left(\bar{p}_{i}, \rho_{i} / 2\right)$. Then the ball $B(y, r / 2)$ lies in the domain $D=M-\cup B\left(\bar{p}_{i}, \rho_{i} / 4\right)$ and by Lemma $4.3 \mathrm{~b}$ the energy in $D$ is $E(D) \leq \varepsilon_{0}$. Hence we can apply Theorem 1.1a (with $r_{0}=0$ and $r_{1}=r / 4$ ) to obtain

$$
\left|F_{A}(y)\right|^{2} \leq \frac{c_{6}}{r^{4}} \int_{D}|F|^{2} .
$$

Now each component of $\partial D$ is a sphere $S_{i}=\partial B\left(\bar{p}_{i}, \rho_{i} / 4\right)$ on which we have, by Lemma $4.3 \mathrm{~cd}$

$$
\sup _{S_{i}}\left|F_{A}\right| \leq c_{1} \frac{\lambda_{i}^{2}}{\rho_{i}^{4}} \sqrt{\delta_{0}} .
$$

If we replace the metric $g$ by the conformal metric $g^{\prime}=16 \rho_{i}^{-2} g$ then $\left(S_{i}, g^{\prime}\right)$ is a sphere that is uniformly $C^{2}$-close the the unit 3-sphere whenever $\varepsilon<\varepsilon_{2}$. Thus (4.15) becomes

$$
\sup _{S_{i}}\left|F_{A}\right|_{g^{\prime}} \leq c_{1} \frac{\lambda_{i}^{2}}{\rho_{i}^{2}} \sqrt{\delta_{0}} \leq c_{7} \sqrt{\delta_{0}}
$$

and hence

$$
\int_{S_{i}}|F|_{g^{\prime}}^{2} d v_{g^{\prime}} \leq c_{8} \varepsilon_{0}
$$

After making $\varepsilon_{0}$ smaller if necessary we can then repeat the argument of equations $(2.7)-(2.9)$, learning that there exists a gauge over $\left(S_{i}, g^{\prime}\right)$ for which the Chern-Simons form (2.4) (which does not depend on the metric) satisfies (using (4.16))

$$
\left|J_{i}\right| \leq c_{9} \int_{S_{i}}|F|_{g^{\prime}}^{2} d v_{g^{\prime}} \leq c_{10}\left(\frac{\lambda_{i}}{\rho_{i}}\right)^{4} \delta_{0}
$$

Hence, returning to the original metric, we can apply (2.3) to (4.14), obtaining

$$
\left|F_{A}(y)\right|^{2} \leq \frac{c_{6}}{r^{4}} \sum_{i}\left|J_{i}\right| \leq \frac{c_{11}}{r^{4}} \sum_{i}\left(\frac{\lambda_{i}}{\rho_{i}}\right)^{4} \delta_{0} .
$$

We next extend Proposition 4.5 to the case where the 'background charge' $k_{0}$ is non-zero. In this case there are problems in choosing the concentration data. For example, suppose $A$ is a $k=2$ instanton consisting of two bumps of energy nearly 1 separated by a small distance; specifically 
suppose there is energy exactly $1-\delta_{0}$ in balls $B(x, \varepsilon)$ and $B\left(y, \varepsilon^{\prime}\right)$ and that $\operatorname{dist}(x, y)=31\left(\varepsilon+\varepsilon^{\prime}\right)$. Then we cannot say that $A$ is $\varepsilon$-concentrated with data $\left\{(x, \varepsilon, \rho=32 \varepsilon, k=1),\left(y, \varepsilon^{\prime}, \rho^{\prime}=32 \varepsilon^{\prime}, k=1\right)\right\}$ because these balls are not disjoint. The alternative is to think of $A$ as a $k=1$ bump superimposed on a $k=1$ background, and use either the data $(x, \varepsilon, \rho=32 \varepsilon, 1)$ or $\left(y, \varepsilon^{\prime}, \rho^{\prime}, 1\right)$. There is, a priori, no reason to prefer one of these descriptions over the other. However, the estimates on $\left|F_{A}\right|$ work out nicely if wre use the smallest concentration data, according to the following definition.

Definition 4.6. A set of concentration data $\left.\left\{p_{i}, \varepsilon_{i}, \rho_{i}, k_{i}\right)\right\}$ is minimal if for no other set of concentration data $\left.\left\{p_{j}^{\prime}, \varepsilon_{j}^{\prime}, \rho_{j}^{\prime}, k_{j}^{\prime}\right)\right\}$ do we have $\min _{j}\left(\rho_{j}^{\prime}\right)<$ $\frac{1}{2} \min _{i}\left(\rho_{i}\right)$.

It is not hard to show that, for a minimal configuration, $\lambda_{\min } \leq \rho_{\min } \leq$ $512 \lambda_{\min }$, where $\lambda_{\min }=\min _{i} \lambda_{i}$. Hence in this case the number $\rho_{\min }=$ $\min _{i}\left(\rho_{i}\right)$ records the scale of the smallest bump in the energy density. Thus the configuration is minimal if it explicitly isolates the smallest bump(s). In the viewpoint of section 5 below, there is evidence that $\rho_{\min }$ is uniformly equivalent to the distance to the boundary of moduli space with its $L^{2}$ Riemannian metric (the shortest path to the boundary is one where the smallest bump bubbles off); the equivalence was proven in [GP2] for $k=1$. Because sup $\left|F_{A}\right| \rightarrow \infty$ as $A$ approaches the boundary of moduli space, this distance is the natural parameter for bounds on $\left|F_{A}\right|$. With this in mind, the following proposition is a counterpart of Proposition 4.5 when $k_{0}>0$.

Proposition 4.7. There exists $C>0$ such that for any $A$ with minimal concentration data $\left\{\left(p_{i}, \varepsilon_{i}, \rho_{i}, k_{i}\right)\right\}$, we have

$$
\left|F^{A}(y)\right| \leq \frac{C}{\rho_{\min }^{2}} \quad \forall y \in M-\bigcup_{i} B\left(p_{i}, \rho_{i} / 2\right) .
$$

Proof. Suppose not. Let $X_{n}=\bigcup_{i} B\left(p_{n, i}, \rho_{n, i} / 2\right)$. Then there exists a sequence of connections $A_{n} \in \mathcal{M}_{k, j}^{\varepsilon}$ with minimal concentration data $\left\{\left(p_{n, i}, \varepsilon_{n, i}, \rho_{n, i}, k_{n, i}\right)\right\}$, and points $y_{n} \notin X_{n}$ for which $\left|F_{n}\left(y_{n}\right)\right| \geq n^{2} / \rho_{n, \min }^{2}$. We may assume that the maximum of $\left|F_{n}\right|$ on $M-X_{n}$ is achieved at $y_{n}$, and denote this sup by $R_{n}^{-2}$; thus $R_{n} \leq \rho_{n, \min } / n$. Let $k_{0}=k-j$; by hypothesis $k_{0} \geq 1$. Consider the balls $B_{n}:=B\left(y_{n}, \rho_{n, \min } / 2\right)$; note that each such ball contains energy at most $k_{0}+k \delta_{0}<k_{0}+1$. In the metric $g_{n}^{\prime}=R_{n}^{-2} g, B_{n}$ has radius at least $n / 2$, and the metrics $g_{n}^{\prime}$ converge uniformly to the flat metric on any compact subset of $\mathbb{R}^{4}$ (using $g_{n}^{\prime}$-normal coordinates centered at $y_{n}$ ). 
Taking a further subsequence, as in the proof of Proposition 4.4 we obtain a sequence of translated, dilated connections $A_{n}^{\prime}$ that converges, uniformly in $C^{1}$ on compact sets, to a finite-energy SD/ASD connection $A_{\infty}^{\prime}$ on $\mathbb{R}^{4}$. By stereographic projection and the Removable Singularities Theorem [U], $A_{\infty}^{\prime}$ extends to $S^{4}$, with finite energy $k^{\prime} \in \mathbb{Z}$, smaller than $k_{0}+1$. Since the connections $A_{n}^{\prime}$ have $\left|F_{n}^{\prime}(0)\right|_{g_{n}^{\prime}}=1$, the limit connection is not flat, so $1 \leq k^{\prime} \leq k_{0}$. Since the convergence is uniform, there is a $L$ large enough that $B\left(y_{n}, L\right)$ contains $A_{n}$-energy $\geq k^{\prime}-\delta_{0}$ for $n$ sufficiently large; in the original metric $g$, this is the ball $B\left(y_{n}, L R_{n}\right)$. Hence there exist numbers $c_{n} \leq L R_{n} / \rho_{n, \min } \leq L / n$ such that $B\left(y_{n}, c_{n} \rho_{n, \min }\right)$ contains $A_{n}$-energy exactly $k^{\prime}-\delta_{0}$. Let $\varepsilon_{n}{ }^{\prime}=c_{n} \rho_{n, \min }$ and $\rho_{n}{ }^{\prime}=32 \varepsilon_{n}{ }^{\prime}$. The $g$-ball $B\left(y_{n}, \rho_{n}{ }^{\prime}\right)$ has $g_{n}^{\prime}$-radius $\leq 32 L$, and hence the convergence of $\left\{A_{n}^{\prime}\right\}$ is uniform on this ball. Since the limit energy is $k^{\prime}, B(0,32 L) \subset \mathbb{R}^{4}$ eventually contains $A_{n}^{\prime}$ energy $<k^{\prime}$, so $B\left(y_{n}, \rho_{n}^{\prime}\right) \subset M$ contains $A_{n}$-energy $<k^{\prime}$. Therefore the data $\left(y_{n}, \varepsilon_{n}{ }^{\prime}, \rho_{n}{ }^{\prime}, k^{\prime}\right)$ satisfy conditions (4.1) and (4.2) of Definition 4.1.

But $\rho_{n}{ }^{\prime} / \rho_{n, \min } \leq 32 L / n \rightarrow 0$, so for large enough $n$ we can replace one of the sets $\left(p_{n, i}, \varepsilon_{n, i}, \rho_{n, i}, k_{n, i}\right)$ by $\left(y_{n}, \varepsilon_{n}{ }^{\prime}, \rho_{n}{ }^{\prime}, k^{\prime}\right)$ to obtain a set of concentration data with $\rho_{\min }$ smaller, contradicting minimality.

The results of this section yield global bounds for connections near the "bottom stratum" of the compactified moduli space $\overline{\mathcal{M}}$. Recall that the bottom stratum is the stratum of $\overline{\mathcal{M}}$ with the maximum number of bubble points; it consists of formal conections whose energy density is a sum of mass $1 \delta$-functions at points $p_{1}, \ldots, p_{k} \in M$. The argument used to prove Proposition 4.2 shows that there is a neighborhood of the bottom stratum consisting of $\varepsilon$-concentrated connections with $k_{0}=0$ and $k_{i}=1$ for all $i$. For such connections Propositions 4.4 and 4.6 both apply, giving the following bound.

Theorem 4.8. There are constants $C, \varepsilon_{3}>0$ such that any $\varepsilon_{3}$-concentrated $S D / A S D$ connection $A$ with concentration data for which $k_{0}=0$ and $k_{i}=$ $1 \forall i$ satisfies

$$
|F(y)| \leq \begin{cases}C \frac{\lambda_{i}^{2}}{\left(\lambda_{i}^{2}+r_{i}^{2}\right)^{2}} & \text { on each } B\left(\bar{p}_{i}, \rho_{i}\right) \\ C \sqrt{\delta_{0}} r^{-2} \sum_{i} \frac{\lambda_{i}^{2}}{\rho_{i}^{2}} & \text { on } M-\cup B\left(\bar{p}_{i}, \rho_{i} / 2\right) .\end{cases}
$$

again with $r_{i}=\operatorname{dist}\left(\bar{p}_{i}, \cdot\right)$ and $r=\min _{i} r_{i}$. 


\section{Applications to the geometry of moduli space.}

We now specialize the results of section 4 to $k=1 \mathrm{SD} / \mathrm{ASD}$ connections. The resulting sharp decay bound translates into a precise statement about the geometry of the $L^{2}$ metric on the boundary of the $k=1$ moduli space. This improves the main results in [G2] and facilitates the proofs in [G3]. Since $k=1$ here we will simply call a connection "concentrated at $p$ " if there is a set of concentration data as in Definition 4.1 with center point $p$; we then define $\bar{p}, \lambda$ as in $\S 4$.

To start, we note that when $k=1$ Theorem 4.7 yields the following particularly simple bound.

Proposition 5.1. There are constants $C, \varepsilon_{3}>0$ such that any $\varepsilon_{3}$-concentrated $S D / A S D k=1$ connection $A$ concentrated at $p$ has, for all $y \in M$,

$$
\left|F_{A}(y)\right| \leq C \frac{\lambda^{2}}{\left(\lambda^{2}+r^{2}\right)^{2}} \quad \text { where } r=\operatorname{dist}(\bar{p}, y) .
$$

Proof. We can apply Theorem 4.7. In fact, if $A$ is $\varepsilon_{3}$-concentrated and $\rho_{1}>0$ then it is still $\varepsilon_{3}$-concentrated with $\rho_{1}$ equal to the injectivity radius $\rho$ (cf. Definition 4.1). Hence the first bound in Theorem 4.7 shows that (5.1) holds on $B(\bar{p}, \rho / 2)$. On the other hand, we always have $r<c_{M} \rho$ where $c_{M}$ is the diameter of $M$ divided by $\rho$, so when $y \notin B(\bar{p}, \rho / 2)$ the second bound in Theorem 4.7 gives

$$
\left|F_{A}(y)\right| \leq \frac{C}{r^{2}} \frac{\lambda^{2}}{\rho^{2}} \leq C c_{M}^{2} \frac{\lambda^{2}}{r^{4}} \leq C c_{M}^{2} \frac{4 \lambda^{2}}{\left(\lambda^{2}+r^{2}\right)^{2}}
$$

For our applications we must revise Proposition 5.1 slightly. In (5.1) $\bar{p}$ and $\lambda$ are the center and scale defined by (4.4) and (4.5). These are somewhat different from the center and scale defined by Donaldson [D]. Roughly speaking, Donaldson's scale $\lambda_{D}(A)$ is defined as the minimal radius of a ball $B$ for which $\int_{B} f_{A}=1 / 2$. For $A$ sufficiently concentrated this ball $B$ is unique, and Donaldson defines its center $\bar{p}_{D}(A)$ to be the "center of $A$ " (for more precise definitions see [D] or [GP2]). The following lemma shows that the distinction between $\bar{p}$ and $\bar{p}_{D}$ and $\lambda$ and $\lambda_{D}$ is essentially immaterial. 
Lemma 5.2. For $k=1 S U(2) S D / A S D$ connections there are positive constants $c_{1}, c_{2}, c_{3}, \varepsilon_{3}$ such that if $A$ is $\varepsilon_{3}$-concentrated at $p$ then

$$
c_{1} \lambda_{D}<\lambda<c_{2} \lambda_{D} \quad \text { and } \quad \operatorname{dist}\left(\bar{p}, \bar{p}_{D}\right) \leq c_{3} \lambda_{D} .
$$

Hence Proposition 5.1 holds (with a different constant) with $\bar{p}$ and $\lambda$ replaced by Donaldson's center $\bar{p}_{D}$ and scale $\lambda_{D}$.

Proof. Donaldson showed (see [D] and $\S 3$ of [GP2]) that for each $\delta_{0}>0$ there are constants $N \geq 1$ and $\lambda_{0}>0$ such that any $k=1$ SD/ASD connection $A$ with $\lambda_{D}<\lambda_{0}$ satisfies

$$
\int_{M-B\left(\bar{p}_{D}, N \lambda_{D} / 32\right)} f_{A}<\delta_{0}
$$

and

$$
\sup _{B\left(\bar{p}_{D}, N \lambda_{D}\right)}|| F_{A}\left|-\frac{4 \sqrt{3} \lambda_{D}^{2}}{\left(\lambda_{D}^{2}+r^{2}\right)^{2}}\right| \leq \lambda_{D}^{-2}
$$

where $r=\operatorname{dist}\left(\bar{p}_{D}, y\right)$. Taking $\delta_{0}$ as in Definition 4.1, (5.3) implies that $A$ is $\varepsilon_{3}$-concentrated with $i=1$ and $p_{i}=\bar{p}_{D}$ and $32 \varepsilon_{i} \leq \rho_{i}=N \lambda_{D}$. Lemma $4.3 \mathrm{c}$ then implies that $\lambda \leq N \lambda_{D} / 12:=c_{2} \lambda_{D}$ and dist $\left(\bar{p}_{D}, \bar{p}\right) \leq N \lambda_{D} / 24:=$ $c_{3} \lambda_{D}$. Note that $B\left(\bar{p}, \frac{23}{24} N \lambda_{D}\right) \subset B\left(\bar{p}_{D}, N \lambda_{D}\right)$, on which (5.4) implies $\left|F_{A}\right| \leq$ $c_{4} \lambda_{D}^{-2}$. Hence for $t<23 N / 24$ we have $\int_{B\left(\bar{p}, t \lambda_{D}\right)} f_{A} \leq\left(c_{4} \lambda_{D}^{-2}\right)^{2} c_{5}\left(t \lambda_{D}\right)^{4}=$ $c_{6} t^{4}$, which is smaller than $1-\delta_{0}$ for $t=c_{1}$ sufficiently small. But then (4.5) implies $c_{1} \lambda_{D} \leq \lambda$, completing the proof of (5.2). But from (5.2) we have $\operatorname{dist}\left(\bar{p}_{D}, \cdot\right)^{2}+\lambda_{D}^{2} \leq \operatorname{const}\left(\operatorname{dist}(\bar{p}, \cdot)^{2}+\lambda^{2}\right)$, so from (5.1) and (5.2) we have $\left|F_{A}(y)\right| \leq C \lambda_{D}^{2} /\left(\lambda_{D}^{2}+r^{2}\right)^{2}$.

Henceforth we will restrict attention to $k=1 S U(2)$ connections, and when we write $\lambda$ we will mean Donaldson's scale.

One consequence of Lemma 5.2 is the sharpening of [G2] concerning the geometry of certain moduli spaces $\mathcal{M}$. To state this theorem, first recall that the $L^{2}$ inner product of differential forms induces a metric $\mathrm{g}$ on $\mathcal{M}$ known as the " $L^{2}$ metric". Specifically, the tangent space to $\mathcal{M}$ at a smooth point $[A]$ (the gauge orbit of a connection $A$ ) is naturally identified with the harmonic space $\mathcal{H}_{A}=\left\{X \in \Omega^{1}(A d P) \mid d_{A}^{-} X=d_{A}^{*} X=0\right\}$. An $A d$-invariant inner product on the Lie algebra of $G$ induces a gauge invariant inner product on 
sections of $A d P \otimes T^{*} M$, which we can integrate to obtain an inner product on $T_{[A]} \mathcal{M}$ :

$$
\mathrm{g}_{[A]}([X],[Y])=\frac{1}{4 \pi^{2}} \int_{M}(X, Y) d v .
$$

Such $L^{2}$ metrics naturally appear in other contexts; for example in Teichmüller theory the analogous construction gives the Weil-Petersson metric, whose geometry has been extensively studied. In gauge theory the $L^{2}$ metric has additional importance because its associated measure is the one that physicists use for path integrals. Work in recent years has begun to clarify the geometry of the $L^{2}$ metric and its connection with physics ([F], [G1$3],[$ GP1-3]).

The most basic examples are the moduli spaces of $k=1 S U(2)$ instantons on compact simply-connected positive-definite manifolds $(M, g)$. These moduli spaces are 5-dimensional and have a "collar region": for sufficiently small $\lambda_{0}>0$ the set $\mathcal{M}_{\lambda_{0}}$ of $\lambda_{0}$-concentrated connections (cf. definition 4.1) is diffeomorphic to $M \times\left(0, \lambda_{0}\right)$ (mapping an instanton to its center and scale provides the diffeomorphism). By examining this collar region, the authors proved that such moduli spaces have finite diameter and volume ([GP2]; this was generalized to arbitrary $k$ in [F]), and that as $\lambda \rightarrow 0, \mathbf{g} \sim 2 d \lambda^{2}+g$ in a $C^{0}$ sense. As a consequence, the Cauchy completion of $(\mathcal{M}, \mathbf{g})$ is the Donaldson compactification in which the boundary $\partial \mathcal{M}$ is an isometric copy of $M$ attached as ideal instantons of scale zero.

Having understood the $C^{0}$ limit of the metric, one can go on to ask whether the curvature of the $L^{2}$ metric becomes singular at the boundary. The analyses in [GP1] and [G1] showed that for the manifolds $M=S^{4}$ and $M=\mathbf{C P}^{2}$ the curvature extends continuously to the boundary and that the boundary is totally geodesic. One might suspect that these are a consequences of the special symmetric space geometry. However, in [G2] the first author showed that the sectional curvature of the collar of the moduli space is bounded above and below for any (compact, simply connected, oriented, positive-definite) manifold whose curvature satisfies a certain positivity condition.

The results of this paper permit us to go further; they show that the basemanifold curvature hypothesis in [G2] is an artifact, and that the boundedness of the moduli-space curvature is a more general phenomenon. Combining the results of this paper, [G2], and [G3], we have the following sharpened result.

Theorem 5.3. Let $(M, g)$ a compact, simply connected oriented, positivedefinite Riemannian 4-manifold, and let $\mathcal{M}$ be the associated modiuli space 
of $k=1 S U(2)$ instantons. Then

(a) the sectional curvature of the $L^{2}$ metric on $\mathcal{M}_{\lambda_{0}}$ is bounded above and below,

(b) the $L^{2}$ metric extends to a $C^{1}$ metric on the completion $\overline{\mathcal{M}}$, and

(c) the boundary $\partial \mathcal{M}$ is a totally geodesic submanifold of $\overline{\mathcal{M}}$.

Proof. Statement (a) was proved in [G2] under the additional assumption that the curvature of $(M, g)$ satisfies the " $\Lambda_{-}^{2}$ condition" ([G2, Theorem 1.1). The sole use of that condition was that it implied (5.1). But Lemma 5.2 establishes the same fact without the $\Lambda_{-}^{2}$ condition. Lemma 5.2 is used throughout [G3], where statements (b) and (c) are proved.

Statements (a) and (b) above are almost certainly not sharp. It seems likely that the curvature extends continuously to the boundary, and, in fact, that the extension of the metric $\mathrm{g}$ is $C^{2}$ in the right coordinate system. This was shown for $S^{4}$ and $\mathrm{CP}^{2}$ in [G2], but, contrary to what one might expect, continuous curvature does not by itself imply $C^{2}$ metric.

Moreover, while Theorem 5.3 and the above " $C^{2}$ conjecture" deal only with $k=1$ instantons on a restricted set of 4-manifolds, the authors believe that the conclusions hold more generally. For larger $k$, boundary strata of the moduli space $\mathcal{M}_{k}(M)$ are subsets of $\bigsqcup_{1 \leq j \leq k}\left(\mathcal{M}_{k-j} \times \operatorname{Sym}^{j}(M)\right)$, where $\operatorname{Sym}^{j}(M)$ is the open manifold of $j$ distinct points in $M$. For a generic metric on $M$ and under suitable conditions on $j$ and $k$ the entire stratum $\mathcal{M}_{k-j} \times \operatorname{Sym}^{j}(M)$ appears as part of the boundary. In this situation, the proof of Theorem 5.3 suggests that conclusions (a) and (b) still hold (in fact it seems likely that the " $C^{2}$ conjecture" holds), and that (c) also holds in a stratified sense: the statum $\mathcal{M}_{k-j} \times \operatorname{Sym}^{j}(M)$ should be totally geodesic in the $L^{2}$ completion $\overline{\mathcal{M}}$.

\section{Appendix.}

In this appendix we prove Proposition 3.3. The basic point is to construct a harmonic function $h$ that behaves at infinity like the Green's function of the laplacian. For concreteness we keep to dimension 4 throughout, but analogous results hold in any dimension greater than 2 . Before starting we make two comments. 
First, on a Riemannian 4-manifold the Green's function with pole at $x$ is a positive harmonic function with a local expansion

$$
G=\frac{1}{r^{2}}+c_{1} \log r+\cdots
$$

in normal coordinates around $x$. One might guess that the Green's function of an asymptotically flat 4-manifold has such an expansion at infinity; if so we could dispense with Proposition 3.3 and use the Green's function instead. However, there is no such expansion. Instead, the Green's function on a asymptotically flat 4-manifold behaves like $G \sim \gamma(\theta) / r^{2}$ where $\gamma \rightarrow 1$ at infinity. Thus bounds of the form (3.7) are the best that can be obtained.

Second, there is a well-developed theory of weighted Sobolev spaces on asymptotically flat manifolds, but this is useless for proving Proposition 3.3. This is precisely because we are dealing with functions that decay at infinity like the Green's function; the Laplacian is not invertible on the weighted Sobolev space of such functions. We avoid this difficulty by producing the function $h$ by variational methods, and then obtaining a decay rate using the maximum principle.

As in Proposition 3.3, consider domains $\Omega_{R}=\Omega(R, \infty)$ in the asiymptotic end. Let $H_{R}$ be the Hilbert space obtained by completing $C_{0}^{\infty}\left(\Omega_{R}\right)$ with respect to the norm

$$
\|f\|^{2}=\int_{\Omega_{R}}|d f|^{2}+\left(\frac{f}{r}\right)^{2} d v_{g}
$$

Lemma A.1. There exists constants $c_{1}$ and $R_{0}$ such that for all $R \geq R_{0}$

$$
\|f\|^{2} \leq c_{1} \int_{\Omega_{R}}|d f|^{2} d v_{g} \quad \forall f \in H_{R} .
$$

Proof. The metric is uniformly euclidean on the asymptotic end, so it suffices to show (A.1) for the euclidean metric and $f \in C_{0}^{\infty}\left(\Omega_{R}\right)$. Integrating by parts in polar cordinates on $\Omega_{R} \subset \mathbb{R}^{4}$

$$
\begin{aligned}
\int_{\Omega_{R}}\left(\frac{f}{r}\right)^{2} r^{3} d r d \omega & =\int \frac{1}{2} f^{2} \partial_{r}\left(r^{2}\right) d r d \omega=\int \frac{f}{r} \partial_{r} f r^{3} d r d \omega \\
& \leq \frac{1}{2} \int_{\Omega_{R}}\left(\left(\frac{f}{r}\right)^{2}+|d f|^{2}\right) r^{3} d r d \omega
\end{aligned}
$$

Subtracting and rearranging yields (A.1). 
Lemma A.2. For each $R \geq R_{0}$ there is a smooth harmonic function $h$ on $\Omega=\Omega_{R}$ with $h=1 / R^{2}$ on $\partial \Omega$ and $h=O(1 / r)$ at infinity.

Proof. Fix $\beta \in C^{\infty}(\bar{\Omega})$ with $\beta \equiv 1 / R^{2}$ in a neighborhood of $\partial \Omega$ and $\beta(x)=0$ for $|x| \geq 2 R$. Then $\psi=-\Delta \beta$ is smooth with compact support. The Lagrangian

$$
L(f)=\int_{\Omega}|d f|^{2}-2\langle f, \psi\rangle
$$

is a smooth functional on $H_{R}$. By Lemma A.1

$$
\begin{aligned}
L(f) \geq \int_{\Omega}|d f|^{2}-2 c_{1} r^{2} \psi^{2}+\frac{1}{2 c_{1}}\left(\frac{f}{r}\right)^{2} & \geq \int_{\Omega} \frac{1}{2}|d f|^{2}-2 c_{1} \int_{D} r^{2} \psi^{2} \\
& \geq \frac{1}{2 c_{1}}\|f\|^{2}-c_{2},
\end{aligned}
$$

so $L$ is coercive and bounded below. It is also strictly convex and hence weakly lower semicontinuous. By the calculus of variations there is a unique absolute minimum $f_{0}$ of $L$ in $H_{R}$. This minimum is a weak solution of the variational equation $\Delta f_{0}=\psi$, so by standard regularity results ([GT] Chapter 6, [M] §5.6) lies in $C^{\infty}(\bar{\Omega})$. Then $h=\beta+f_{0}$ is smooth and harmonic with $h=1 / R^{2}$ on $\partial \Omega_{R}$. Finally, at each point $x$ with $|x| \geq 4 R$ the ball $B=B(x, r / 2)$ lies in the region $\Omega(2 R, \infty)$ where $\beta=0$. We then get a pointwise bound by applying [GT] Theorem 9.20 on $B$ to get

$$
|h(x)| \leq c_{3}\left(\frac{1}{r^{4}} \int_{B} h^{2}\right)^{1 / 2} \leq \frac{4 c_{3}}{r}\left(\int_{B}\left(\frac{f}{r}\right)^{2}\right)^{1 / 2} \leq \frac{4 c_{3}}{r}\left\|f_{0}\right\|
$$

and noting that $\left\|f_{0}\right\|^{2} \leq 2 c_{1} L\left(f_{0}\right)+c_{2}$ with $L\left(f_{0}\right) \leq L(0)=0$ by (A.2) and the fact that $f_{0}$ minimizes $L$.

We next use the maximum principle to get a better decay rate on $h$. First note that on an asymptotically flat manifold the formula for the Laplacian $\Delta=-\frac{1}{\sqrt{g}} \partial_{i} \sqrt{g} g^{i j} \partial_{j}$ shows that

$$
\Delta r^{\alpha}=d^{*} d r^{\alpha}=-\alpha(\alpha+2) r^{\alpha-2}+\phi_{\alpha}
$$

where $\phi_{\alpha}=O\left(r^{\alpha-4}\right)$ and $d \phi_{\alpha}=O\left(r^{\alpha-5}\right)$. In particular, $\left|\phi_{-2}\right| \leq c_{4} r^{-6}$ and $\left|\phi_{-3}\right| \leq c_{5} r^{-7}$ on $\Omega\left(R_{0}, \infty\right)$. 
Lemma A.3. For each $0 \leq \varepsilon \leq 1$ there is a $R_{\varepsilon}$ such that for each $R \geq R_{\varepsilon}$ the function $h$ obtained in Lemma A.2 satisfies

$$
\frac{1-\varepsilon}{r^{2}} \leq h \leq \frac{1+\varepsilon}{r^{2}}
$$

and if $\varepsilon$ is sufficiently small then we also have $|d h| \leq r^{-3}$.

Proof. Fix $\varepsilon$ and set $R_{\varepsilon}=\max \left\{R_{0}, \sqrt{\left(c_{4} / \varepsilon+c_{5}\right.}\right\}$. Then the functions

$$
f_{ \pm}=\frac{(1 \mp \varepsilon)}{r^{2}} \pm \frac{\varepsilon R}{r^{3}} \text {. }
$$

satisfy $f_{+}=f_{-}=R^{-2}$. Hence for $r \geq R \geq R_{\varepsilon}$ (A.3), the decay $h==O(1 / r)$, and our choice of $R_{\varepsilon}$ give

$$
\begin{aligned}
\Delta\left(h-f_{-}\right) & =-(1+\varepsilon) \Delta r^{-2}+\varepsilon R \Delta r^{-3} \\
& \leq(1+\varepsilon) c_{4} r^{-6}+\varepsilon R\left(-3 r^{-5}+c_{5} r^{-7}\right) \\
& \leq r^{-6}\left(2 c_{4}+\varepsilon c_{5}-3 \varepsilon R_{\varepsilon}^{2}\right) \\
& \leq 0
\end{aligned}
$$

Hence $h \leq f_{-} \leq(1+\varepsilon) / r^{2}$ by the maximum principle. Similarly, one finds that $\Delta\left(f_{+}-h\right) \leq 0$ for the same $R_{\varepsilon}$, so $h \geq f_{+} \geq(1-\varepsilon) / r^{2}$.

Now write $h=1 / r^{2}+\xi$. Since $h$ is harmonic $\Delta \xi=-\Delta r^{-2}=\phi$ where $|d \phi| \leq c_{6} r^{-7}$, and $|\xi|=\left|h-1 / r^{2}\right| \leq \varepsilon / r^{2}$ by (A.4). Applying [GT] T.heorems 6.2 and 6.6 on $\Omega=\Omega(R, \infty)$

$$
|d \xi|_{\Omega} \leq \frac{c_{7}}{r} \sup _{\Omega}\left(|\xi|+r^{2}|d \phi|\right) \leq \frac{c_{7}}{r}\left(\frac{\varepsilon}{r^{2}}+c_{6} r^{-4}\right) .
$$

Now take $\varepsilon \leq 1 / 4 c_{7}$ and make $R_{\varepsilon}$ larger is necessary to ensure that $R_{\varepsilon}^{2} \geq$ $4 c_{6} c_{7}$ and that $|d r| \geq 3 / 4$ on $\Omega\left(R_{\varepsilon}, \infty\right)$. Then for all $r \geq 2 R$,

$$
|d h|=\left|d r^{-2}+d \xi\right| \geq \frac{2|d r|}{r^{3}}-|d \xi| \geq \frac{1}{r^{3}} .
$$

Proposition 3.3 follows from Lemma A.3 by fixing $\varepsilon \leq 1 / 4$ and noting that

$$
\xi=\frac{1}{4} h^{-2}|d h|^{2} \geq \frac{1}{4} h\left(\frac{1+\varepsilon}{r^{2}}\right)^{-3} \frac{1}{r^{6}} \geq \frac{1}{8} h .
$$




\section{References.}

[D] S. K. Donaldson, An application of gauge theory to four-dimensional topology, J. Diff. Geom. 18 (1983), 279-315.

[DK] and P. Kronheimer, The Geometry of Four-Manifolds, Oxford University Press, New York (1990).

[F] P. Feehan, Geometry of the ends of the moduli space of anti-self-dual connections over negative definite four-manifolds, J. Diff. Geom., to appear.

[FU] D.S. Freed and K.K Uhlenbeck, Instantons and Four-Manifolds, SpringerVerlag, New York (1984).

[G1] D. Groisser, The geometry of the moduli space of $\mathbf{C P}^{2}$ instantons, Inventiones Mathematicae, 99 (1990), 393-409.

[G2] D. Groisser, Curvature of Yang-Mills moduli spaces near the boundary, I, Commun. Anal. and Geom. 1 139-216, (1993).

[G3] Curvature of Yang-Mills moduli spaces near the boundary, II: Totally geodesic boundary, Preprint (1995).

[GP1] D. Groisser and T. H. Parker, The Riemannian geometry of the Yang-Mills moduli space, Commun. Math. Phys. 112 (1987), 663-689.

[GP2] The geometry of the Yang-Mills moduli space for definite manifolds, J. Diff. Geom. 29 (1989).

[GP3] Semiclassical Yang-Mills Theory I: Instantons, Commun. Math. Phys. 135 (1990), 101-140.

[GT] D. Gilbarg and N. S. Trudinger, Elliptic Partial Differential Equations of Second Order, second edition, Springer-Verlag, Berlin, New York (1983).

[M] C. B. Morrey, Jr., Multiple Integrals in the Calculus of Variations, Springer-Verlag, Berlin, Heidelberg (1966).

[R] J. Røade, Decay estimates for Yang-Mills fields; two new proofs, in Global Analysis in Modern Geometry, Publish or Perish, Houston (1993).

[U] K. K. Uhlenbeck, Removable singularities in Yang-Mills fields, Commun. Math. Phys. 83 (1982), 11-30.

Received August 25, 1995. 
UNIVERSITY OF FLORIDA

DEPARTMENT OF MATHEMATICS

GAINESVILle, FL 32611

E-mail address: GROISSER@MATH.UFL.EDU

AND

Michigan State University

DEPARTMENT OF MATHEMATICS

EAST LANSING, MI 48824

E-mail address: PARKER@MATH.MSU.EDU 